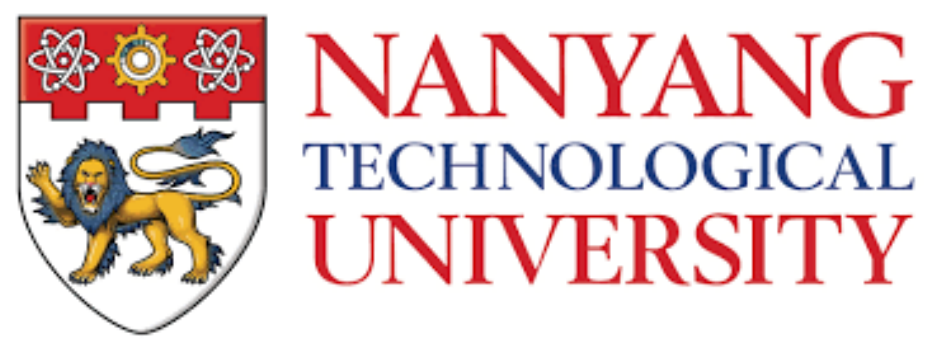

EXPERIMENTAL STUDY OF ROBOTIC POLISHING PLANNING FOR UNEVEN SURFACES WITH FORCE CONTROL

HONG JIE

School of Electrical \& Electronic Engineering 2017 



\section{EXPERIMENTAL STUDY OF ROBOTIC POLISHING PLANNING FOR UNEVEN SURFACES WITH FORCE CONTROL}

HONG JIE

School of Electrical \& Electronic Engineering

A thesis submitted to the Nanyang Technological University in partial fulfilment of the requirements for the degree of Master of Engineering 



\section{Abstract}

Polishing technique plays a critical role in industrial manufacturing to smooth the surface of products such as turbine blades, die and mold. To improve the polishing process, many technologies are explored and applied. The automated polishing processes are categorized as computer numerically controlled machine polishing and industrial robotic polishing. The robotic arms possess the special advantages in working envelope, flexibility and production cost against computer numerically controlled machines. In addition, the robotic force control does improve the polishing performance in both surface material removal and surface roughness. This project strives to improve the polishing technologies by using combination of robotic techniques and intelligent computation. A specific polishing task that uneven surfaces should be polished to the required smoothness has been first defined. In order to solve the planning problem for this specific polishing task, the polishing process model is investigated and the polishing parameters are optimized based on computational intelligence. Neural network is employed to formulate the model while genetic algorithm is utilized to search for the best parameters. In experiments, workpiece surfaces under the robotic polishing using the planned parameters successfully achieve the desired polishing performance, which verifies the effectiveness of the proposed solution. 


\section{Acknowledgement}

First and foremost, I would like to thank my supervisor Professor Danwei Wang for providing me with a valuable opportunity to work under his guidance towards my Master of Engineering programme. I have thoroughly enjoyed my project meetings and other interactions with him due to his optimistic and friendly personality. His timely words of advice are definitely helpful to my progress in research and the successful completion of my degree programme.

I would like to express my gratitude to another research group member, Dr. Abd El Khalick Mohammad who gave me a lot of kindly help. Also, I would like to thank Mr. Chia Chiu Kiat of Robotics Laboratory for having provided me with the necessary resources to successfully carry out my research.

Last but definitely not the least, I would greatly thank my parents for their constant support, encouragement and endless love during my master study. 


\section{List of Figures}

1.1.1 MP process is conducted by human labor (Image Courtesy: axiumprocess.com) . . . . . . . . . . . . . . 14

1.1.2 CMP process is conducted by the special equipment (Image Courtesy: levitronix.com) $\ldots \ldots \ldots \ldots$

1.2.1 CNC machine (Image Courtesy: cmz.com) . . . . . . . . . . . 17

1.2.2 CNC polishing (Image Courtesy: matricats.com) _ . . . . . . 17

1.2.3 Robotic polishing process (Image Courtesy: completesurfacefinishing.com $) \ldots \ldots \ldots \ldots \ldots$

1.2.4 Robotic polishing process for the propeller (Image Courtesy: A*STAR) 20

1.2.5 ABB software platform: RobotStudio . . . . . . . . 23

2.1.1 The closed-loop of robotic polishing system . . . . . . . 26

2.1.2 Experimental setup for robotic polishing . . . . . . . . 28

2.1.3 The sectional view of the proposed polishing end-effector . . . . 29

2.1.4 Material removal measurement (the left figure) and roughness measurement (the right figure) during experiments . . . . . . 32 
3.1.1 Uneven surface polishing (a) Unpolished surface with two different heights/roughness (b) Polished surface with the identical height $/$ roughness . . . . . . . . . . . . . 38

3.2.1 The proposed planning algorithm for polishing uneven surface . $\quad 40$

$3.2 .2 \mathrm{NNW}$ architecture $\ldots \ldots \ldots \ldots . \ldots \ldots$

3.2.3 GA planning process with NNW prediction . . . . . . . . 43

4.0.1 The illustration of measurement sampling . . . . . . . . . . 48

4.1.1 The mean square error for the training epochs $\ldots \ldots \ldots 50$

4.3.1 Variation of GA fitness value . . . . . . . . . . . . 56

4.4.1 The experimental results for the $1^{\text {st }}$ surface and the $2^{\text {nd }}$ surface in

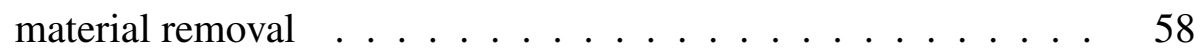

4.4.2 The experimental results for the $1^{\text {st }}$ surface and the $2^{\text {nd }}$ surface in roughness improvement . . . . . . . . . . . 58

4.4.3 The average material removal/roughness improvement for the $1^{\text {st }}$ surface and the $2^{\text {nd }}$ surface $\ldots \ldots \ldots . \ldots 59$ 


\section{List of Tables}

1.1 RAPID instructions from force control module . . . . . . . . 24

3.1 Polishing parameters and polishing performance . . . . . . 39

4.1 Experimental results of NNW training samples . . . . . . . 51

4.2 Experimental results of NNW testing samples . . . . . . 53

4.3 Planned results with desired performance . . . . . . . . 60 


\section{Contents}

$\begin{array}{ll}\text { Abstract } & 1\end{array}$

$\begin{array}{lr}\text { Acknowledgement } & 3\end{array}$

$\begin{array}{ll}\text { List of Figures } & 4\end{array}$

$\begin{array}{ll}\text { List of Tables } & 7\end{array}$

1 Motivation and State-of-the-Art Technology 11

1.1 Conventional Polishing Process . . . . . . . . . . . . . . 12

1.1.1 Mechanical Polishing . . . . . . . . . . . . . 13

1.1.2 Chemical Mechanical Polishing . . . . . . . . . 14

1.2 Automated Polishing Technology . . . . . . . . . . . . . 15

1.2.1 CNC Machine Polishing . . . . . . . . . . 16

1.2.2 Robotic Polishing . . . . . . . . . . . . 18

1.2.3 Force Control for Polishing . . . . . . . . . . 21

2 Research Platform and Project Objectives 25 
2.1 Experimental Robotic Polishing System . . . . . . . . . . 25

2.1.1 ABB Robotic Arm with End-effector . . . . . . . 26

2.1.2 Polishing Performance Measurement . . . . . . . . 30

2.2 Projective Objectives ................. . . 31

3 Process Planning of Uneven Surface Polishing 35

3.1 Robotic Polishing Task for Uneven Surfaces . . . . . . . . . . . 37

3.2 Polishing Planning Procedure . . . . . . . . . . . . . 38

3.2.1 Neural Network Approximation . . . . . . . . . 40

3.2.2 Genetic Algorithm Planning Procedure . . . . . . . . 42

4 Experimental Results $\quad 47$

4.1 Neural Network Training . . . . . . . . . . . . . . . . 47

4.2 Neural Network Testing . . . . . . . . . . . . . . . . . 52

4.3 Genetic Algorithm Optimization . . . . . . . . . . 53

4.4 Planned Parameters and Verification Results . . . . . . . . . . 56

5 Conclusion and Future Work $\quad 61$

5.1 Conclusion ......................... 61

5.2 Future Work . . . . . . . . . . . . . . . . 62

$\begin{array}{lr}\text { Publications } & 64\end{array}$

$\begin{array}{lr}\text { Bibliography } & 65\end{array}$ 


\section{Chapter 1}

\section{Motivation and State-of-the-Art}

\section{Technology}

Polishing has been an important finishing process for a better surface quality. Most industrial metal products made of stainless steel, aluminium alloy or titanium metal usually must meet some strict specifications in surface gloss and surface roughness. Improving the polishing process is highly demanded and has attracted a lot of attention in recent years. In order to realize this objective, the combination of automation and polishing is deemed as a key solution. In this study, it has been discussed that there exist positive effects of robotic approaches on surface polishing. The overall purposes of this research topic can be summarized as follows: (1) To study the improvements for mechanical polishing through robotic technologies; (2) To propose and design an industrial robotic polishing system with a compliant end-effector; (3) To fulfill the process planning of uneven surface pol- 
ishing based on the cooperation between the robotic technique and computational intelligence.

The report is mainly organised as five chapters. Chapter 1 is motivation and state-of-the-art technology. In this chapter, the robotic technologies for polishing are discussed. A robotic experimental setup that comprises a robotic manipulator and a polishing end-effector is then presented for the subsequent research in Chapter 2. Chapter 3 introduces how the process planning is conducted for polishing an uneven surface. The polishing goal to be accomplished is particularly defined and the structure of the planning solution is introduced in this chapter. Accordingly, in Chapter 4, the experimental results validate that the proposed solution to achieve both surface smoothness accuracy and surface position accuracy is effective. Conclusion and future works are following in Chapter 5.

\subsection{Conventional Polishing Process}

Polishing technologies are widely applied in different industries to improve surface texture as producing die, mould, turbine blades, engine vanes and propellers. They are finishing processes for smoothing workpieces' surface by using special tools. Here, we summarize reasons why the products should be finished by the polishing process as follows:

(i) Polishing is often used to improve the surface cleanability of products. It is able to create a more reflective surface that was originally a coarse one. To 
be more specific, it helps to get rid of the original attached contamination and oxidation;

(ii) Polishing can also prevent the workpiece from the corrosion. A smoother surface is less likely to adhere deposits and thus it decreases the risk of chemical corrosion. Meanwhile, a glossier surface has a better performance in the abrasive resistance which is the ability to avoid the mechanical wear to a certain degree.

In terms of the applied energy sources, the conventional polishing technology can be mainly classified into two types: mechanical polishing and chemical mechanical polishing. The following content briefly introduces the core principle and mechanism for these two different polishing methods.

\subsubsection{Mechanical Polishing}

Mechanical polishing (MP) is a fundamental surface polishing process by using only the mechanical motion as energy source. Its basic principle is to physically remove a thin surface layer and therefore achieve a required surface smoothness. To remove the material via compression and friction, the polishing pad with abrasives attached on the polishing tool head should contact the workpiece surface and it is also rotating relative to the workpiece surface. Fig. 1.1.1 shows a case where the MP process is being conducted by an expert worker. Actually, the situation is that nowadays most of MP processes are completed manually. 


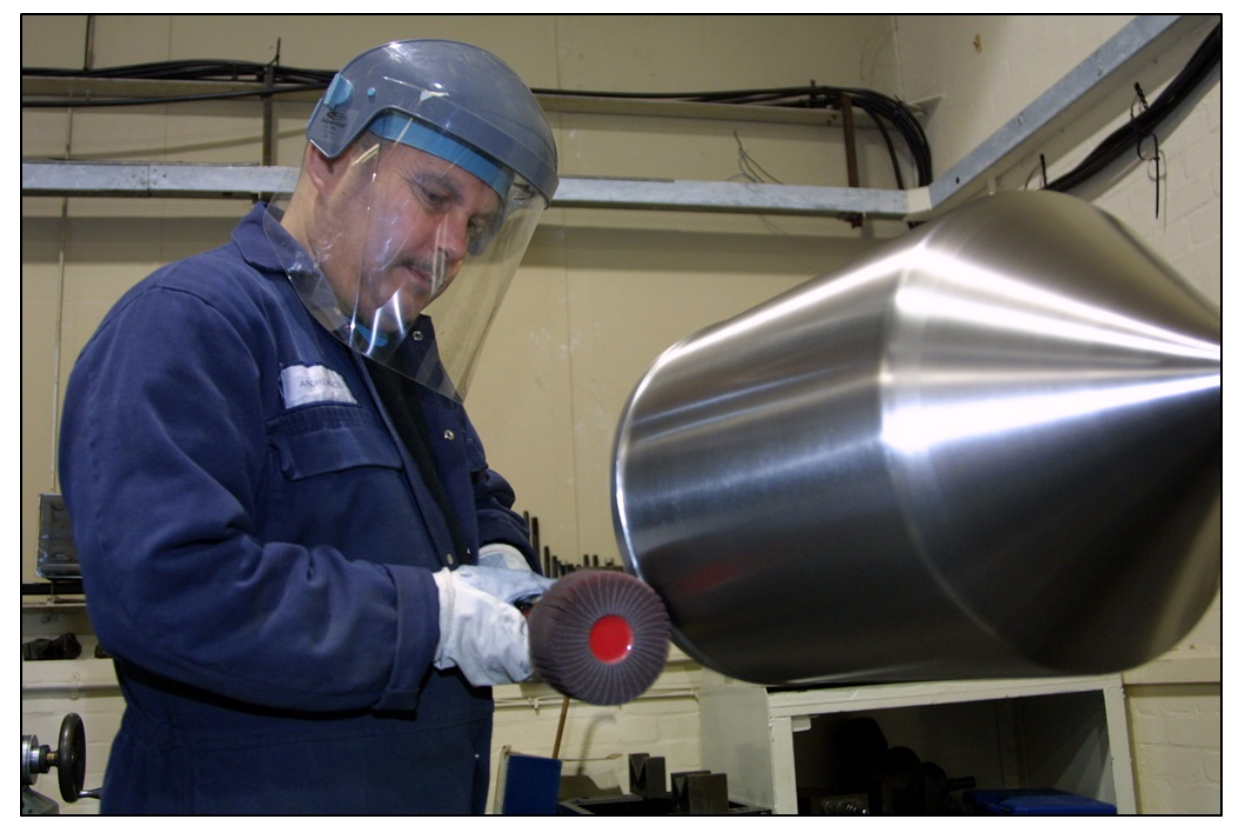

Figure 1.1.1: MP process is conducted by human labor (Image Courtesy: axiumprocess.com)

\subsubsection{Chemical Mechanical Polishing}

Chemical mechanical polishing (CMP) technology uses chemical action in addition to the mechanical action compared to MP. In MP, the mere mechanical motion causes too much collision and hence reduces the tool life. But for CMP, two actions are taking effects synergistically to carry out surface finishing. As it can be seen from Fig. 1.1.2, polishing head and polishing pad are rotating in the opposite direction. The workpiece of small size is inserted at the tip of polishing head. The slurry contains both abrasive particles and corrosive chemicals. It is obvious that the abrasive particles provides indentation for the removal of the surface material while the chemical like nitric acid facilitates the corrosion reactions of the surface material. 


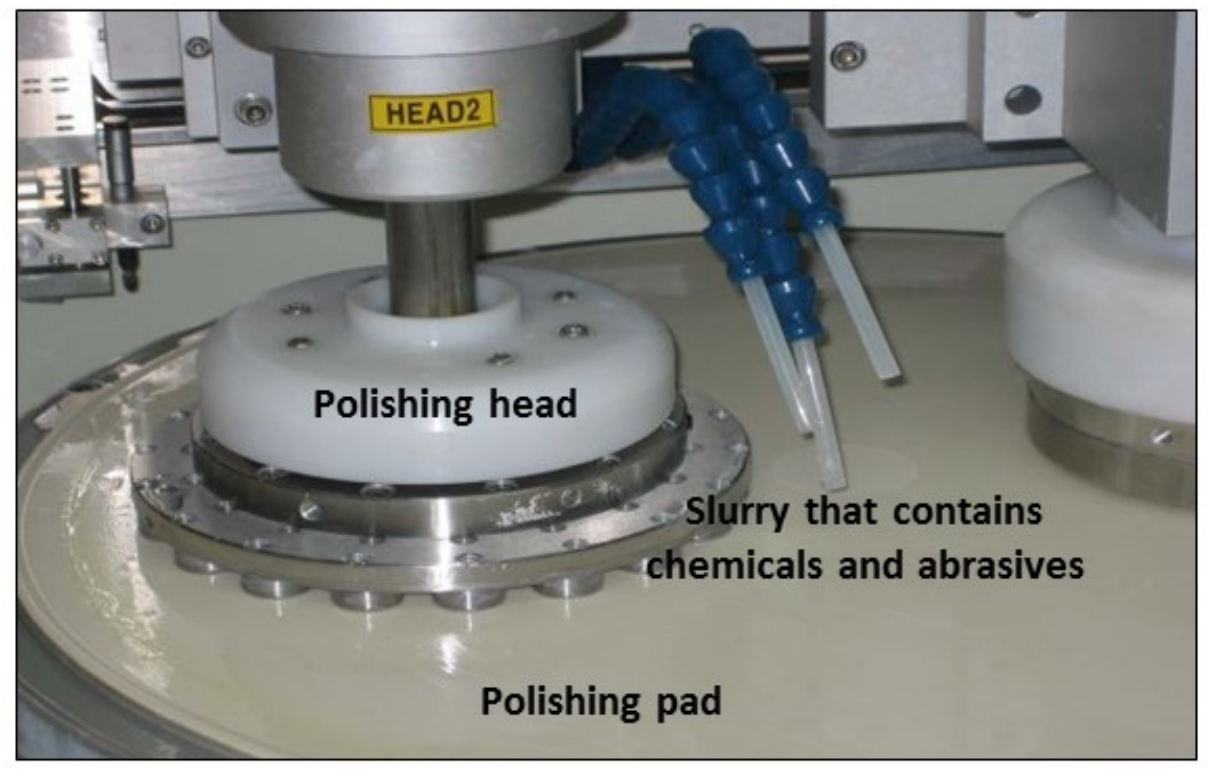

Figure 1.1.2: CMP process is conducted by the special equipment (Image Courtesy: levitronix.com)

\subsection{Automated Polishing Technology}

Nowadays, workpieces to be polished like turbine blades, propellers, etc, become more complex. Polishing these workpieces under only human labor cannot ensure the high machining precision. Besides, the manual polishing normally needs skilled laborers, which means high production cost [1]. Added to this point, the manual polishing is generally carried out in the awkward polishing working condition where the workers suffer metal dust, harmful chemicals and severe noise [2].

With the increasing demand for polishing process, the automatic material removal technologies are presented to replace the human labor. They can be divided into computer numerically controlled (CNC) machines for polishing and robotic 
polishing systems. Researchers are now striving to enhance the level of polishing automation based on these two technologies to substitute machines for labor work.

\subsubsection{CNC Machine Polishing}

CNC machining is a common machining technology that is operated by programming and executing computer codes. The machine automatically follows the commands that is interpreted from computer codes. The installed tools can fulfill a wide range of machining operations like turning, milling, cutting, welding, bending, punching, etc. Figs. 1.2.1 and 1.2.2 depict a $\mathrm{CNC}$ machine and a finishing process carried out by $\mathrm{CNC}$ machine, respectively.

For surface machining, CNC machines generally provide fast and accurate tool movements. Further, CNC tool can be easily positioned and oriented along multi-axis. Benefit from this outstanding mobility, CNC machine is able to finish the workpiece with irregular shapes compared to the conventional equipment. Walker et. al [3] utilize a 7-axis CNC machine to polish spherical surfaces and aspheric surfaces. In [4], the plastic injection mold with free-form surface is burnished by a CNC system.

It should be stressed that the special fixtures are necessary components for CNC machines to accommodate, align and hold tools and workpieces. These fixtures like tool changers, fixture plates, vises and clamps typically require precision machining so as to precisely lay and line up the items. 


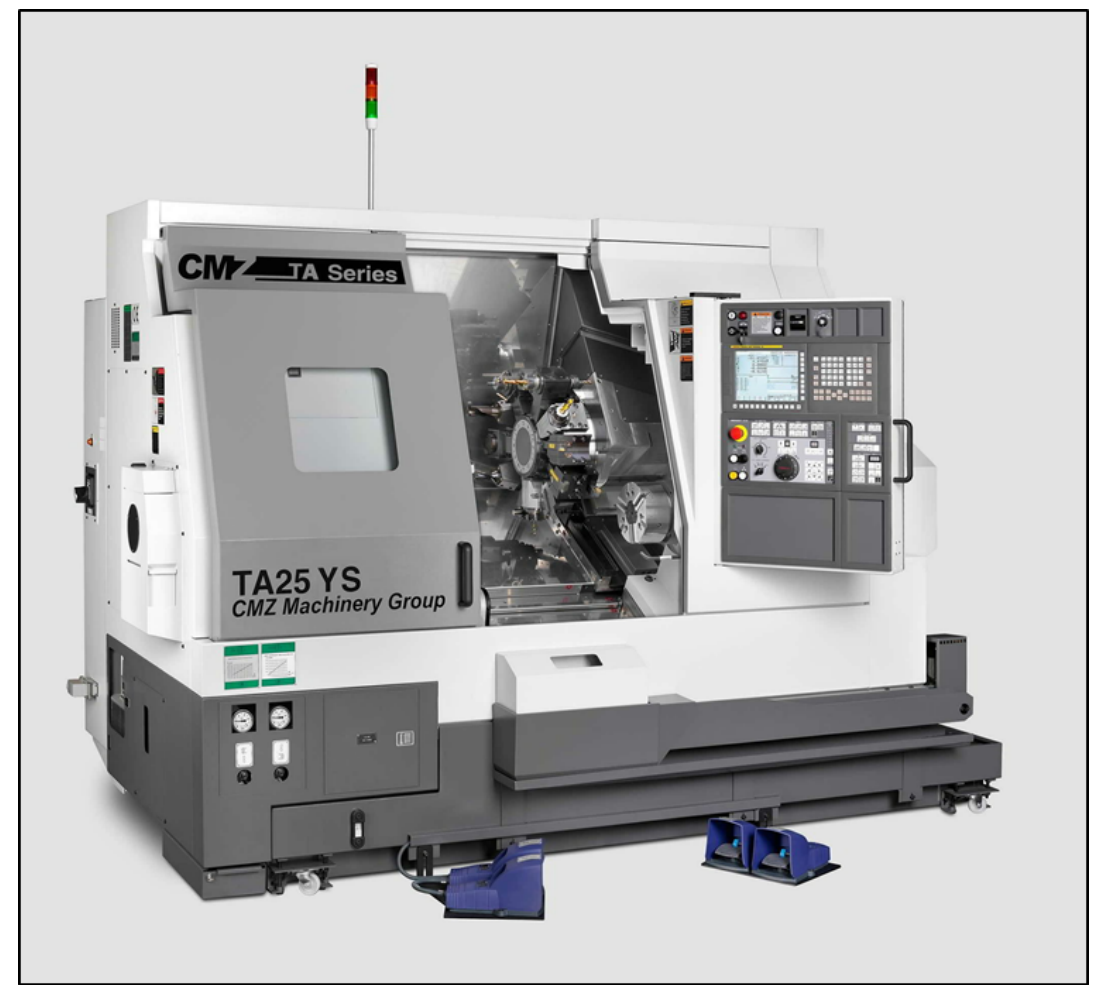

Figure 1.2.1: CNC machine (Image Courtesy: cmz.com)

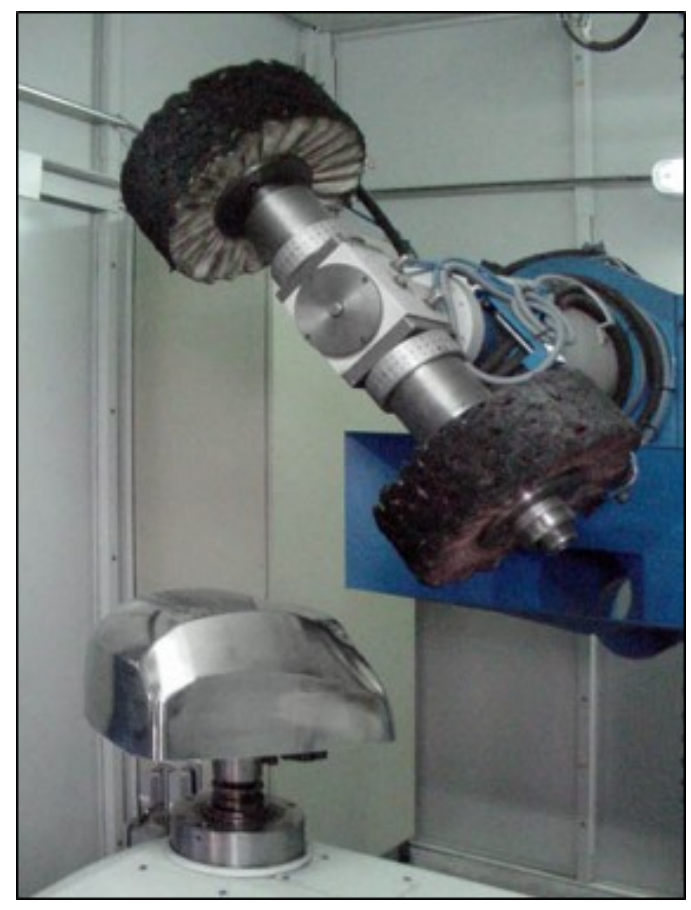

Figure 1.2.2: CNC polishing (Image Courtesy: matricats.com) 


\subsubsection{Robotic Polishing}

The increasing need for manufacturing workpiece with large body and complex surface requires us to improve the conventional polishing in an innovative way. Besides the limitation of traditional manual polishing that has been discussed, the traditional polishing machine also has limitations. For example, CMP equipment shown in Fig. 1.1.2 is commonly applied for polishing flat stainless steel substrate and flat copper wafer. It cannot deal with the workpiece of complex geometry.

The appearance of $\mathrm{CNC}$ machines do automate the polishing process, whereas it is definitely restricted by small available working area [5]. Another disadvantage for $\mathrm{CNC}$ machines is that it usually takes time to design and manufacture the special fixtures that are already introduced. In order to overcome above mentioned problems, robotic polishing, the combination of an industrial robotic arm and a polishing device is certainly viewed as a better choice [6]. Fig. 1.2.3 illustrates how a robotic polishing cell is configured and the reasons why robotic techniques are used are demonstrated in the following paragraphs.

\section{Working Envelope}

The large work envelope of industrial robot is able to cope with the largesize workpiece [7]. However, by using CNC machines, huge workpieces should be split before polishing and reassembled after polishing [8]. The first example in Fig. 1.2.4 shows that only robotic arm, not the CNC machine, can execute 


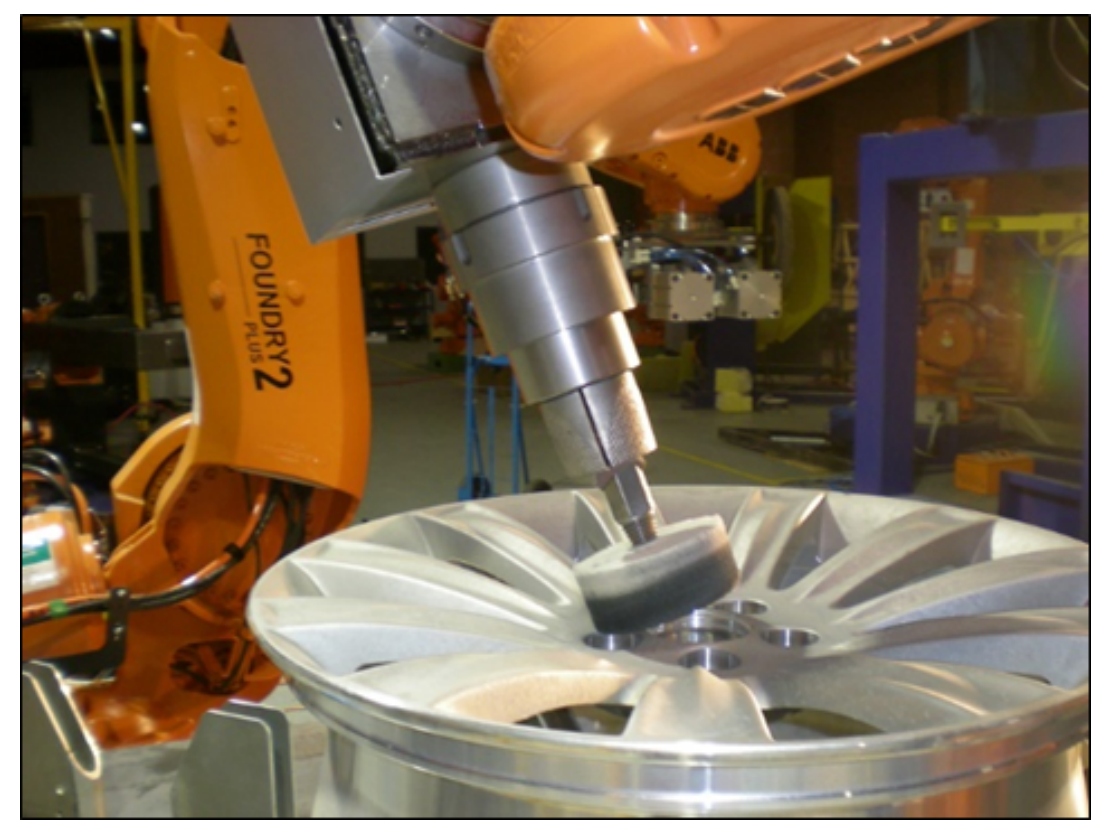

Figure 1.2.3: Robotic polishing process (Image Courtesy: completesurfacefinishing.com)

the polishing task for such a big propeller. Sabourin et. al [9] [10] applied a robotic system to perform polishing of large hydraulic turbines. Huang et. al [11] designed an automatic grinding system, attempting to replace the manual overhaul of turbine vanes in big size.

\section{Flexibility}

The industrial robots' excellent flexibility helps to improve manufacture processes. First of all, articulated robots are easy to be removed and assembled due to their light weight compared to $\mathrm{CNC}$ machines. Hence, the production line can be easily built by cooperative robots to do a wider range of jobs. A multi-purpose cell that contains several robots was proposed in [12] for repairing turbine blades and the whole production chain includes cleaning, inspection, laser cladding, cut- 


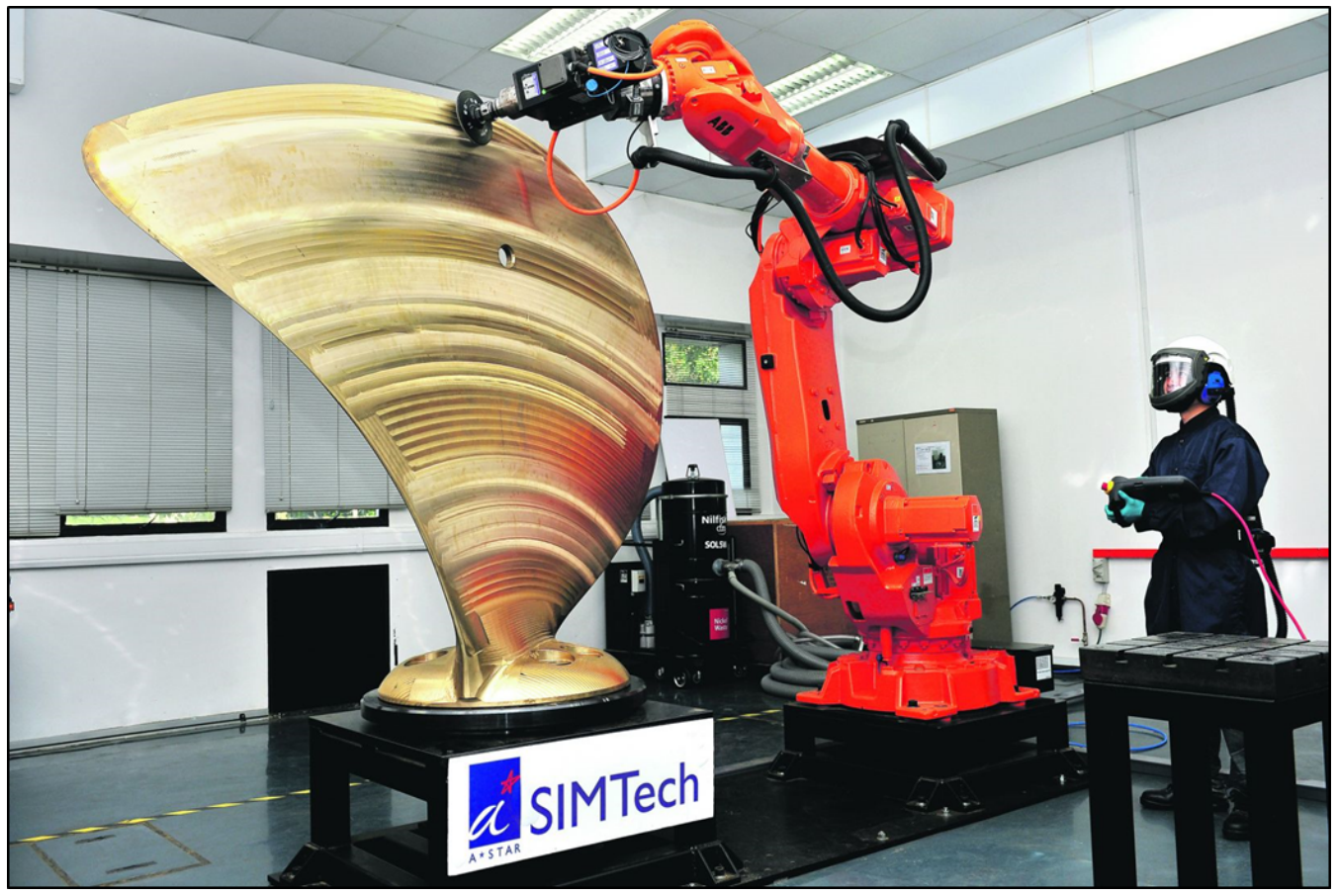

Figure 1.2.4: Robotic polishing process for the propeller (Image Courtesy: A*STAR)

ting and grinding. Differ from robots, CNC machines which are difficult to shift, regularly have heavy weight and complex calibrations.

Another point of the flexibility is that for only one industrial robot, it is applicable to attach different designed end-effectors to perform different material removal processes [13]. Not only the polishing process, but also the necessary machining before polishing like cutting and milling can be operated under merely one robot. Coelho et.al [14] utilize the ball end tool mounted in the articulated arm to conduct milling process and achieve ideal roughness for aluminium alloy. The robotic cutting task was carried out with a cutting tool in [15]. The articulated arm with a handy air-driver sander is applied for sanding process of wooden furniture [16]. Usually for one CNC machine, its tools cannot be interchangeable 
with others of different types. For instance, turning lathe and milling lathe are not allowed to use the same type of machining tool.

The mechanical configurability of $\mathrm{CNC}$ machines that defines how people machine the workpiece is difficult to be changed. In this regard, engineers can redesign this configurability according to their requirements based on the robotic platform and thereby machining procedures become more flexible [6].

Low price is also considered as an important advantage of industrial robots. Unlike CNC machines that are usually costly, the choice of robotic system certainly saves the production expense [14]. As long as the machined surface error of robotic system could comply with manufacturing demands, the solution using robot-assisted machining which is more cost-effective is the better choice. There are still many other robotic polishing applications that have been introduced in the previous literature [17] [18] [19] [20] [21].

\subsubsection{Force Control for Polishing}

Force control is a popular industrial robotic technique where tremendous research works have been already done. The corresponding algorithm is to ensure that the force acting between the robot and the workpiece can be regulated to the desired value as well as the desired direction. For simplicity, a constant exerted force can be kept as the robot is interacting with the workpiece. The most force control for polishing is accomplished by "through-the-arm" approach. This approach means that the robot applies all its joints to exert the force. However, the 
performance of force tracking using this approach would be sometimes unsatisfactory [22]. Therefore, the other way employing "around-the-arm" approach is coming up. It indicates the additional axes are added for the robot by equipping the end-effector with auxiliary motors. Force control in this approach is realized by applying only additional axes of motor.

In this subsection, the discussion is provided upon how the force control can essentially improve the polishing performance. Another covered aspect is that how $\mathrm{ABB}$ force control module can be used in programming to fulfill force regulation under "through-the-arm" approach. This software module facilitates the force control for experiments in Chapter 4.

\section{Improvements}

Material removal rate represents how much volume of material can be removed per unit time. One the one hand, high material removal rate during polishing process is one of the most essential goals researchers are pursing. The higher material removal rate means the manufacturing time can be definitely shortened and thus enhance the working efficiency. As it can be easily understood, more material would be removed once more contact force is applied on the workpiece surface. On the other hand, excessive material removal rate should be avoided to keep the workpiece geometry accuracy [23], which needs force value to be appropriate.

Roughness, the parameter to describe the surface texture, is quantified by an 
average deviation of surface from the ideal level. For roughness, it has been acknowledged that the lower roughness can be achieved under force control. The force could be spread more uniformly over the surface if the force maintained constant [24] [25]. Yet, the achievement of the lower roughness does not mean to generate too much force. Because the heavy strokes resulted from too much force may cause serious pumps and pits on the surface. Sometimes the force acting between polishing tool and polishing pad should be studied and specified.

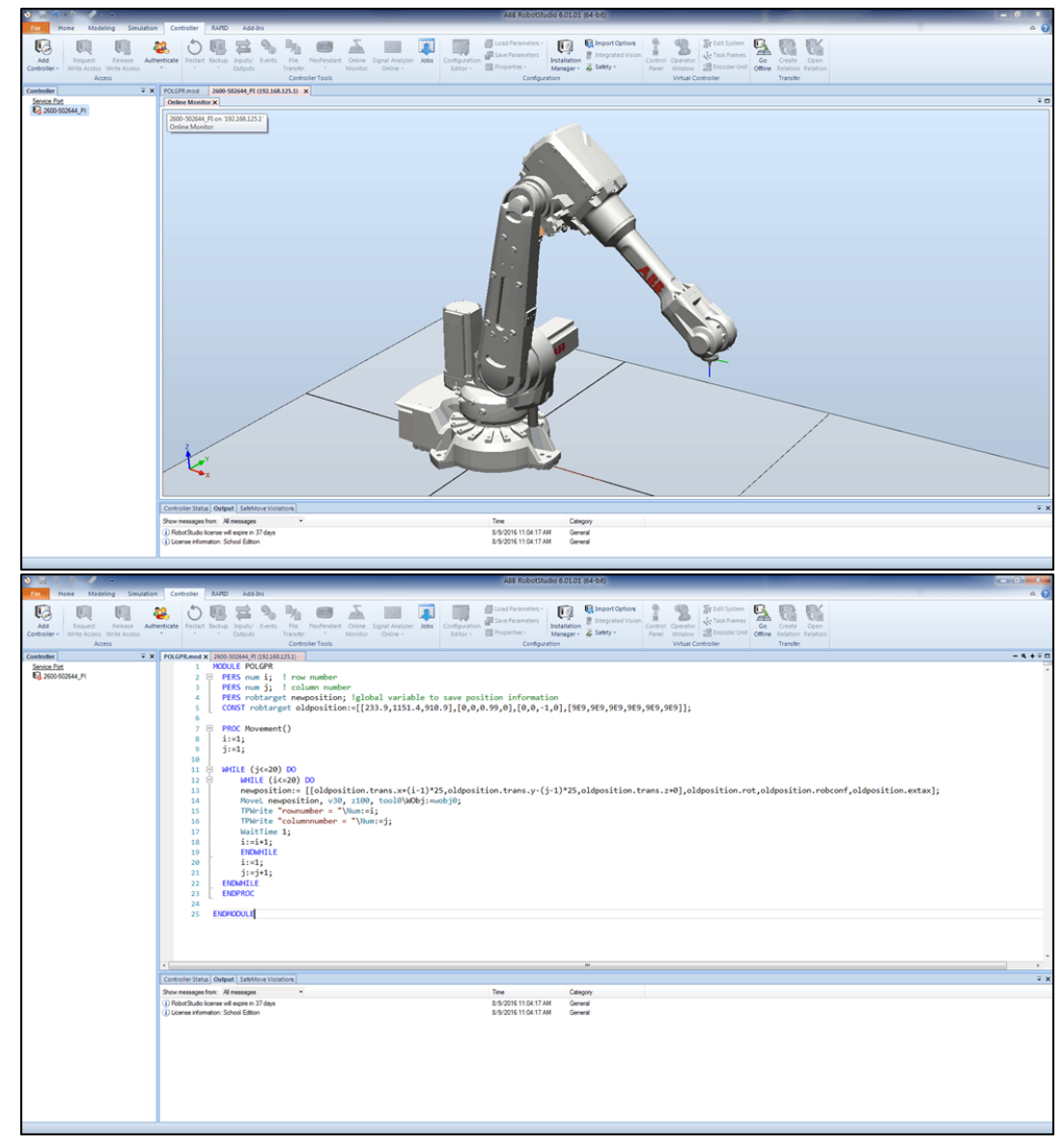

Figure 1.2.5: ABB software platform: RobotStudio 
Table 1.1: RAPID instructions from force control module

\begin{tabular}{l|l}
\hline Instruction & Description \\
\hline FCLoadID & To measure and identify the payload before formal calibration \\
\hline FCCalib & $\begin{array}{l}\text { To calibrate the force sensor and compensate for payload gravity } \\
\text { by using offset information detected from FCLoadID }\end{array}$ \\
\hline FCRefForce & To define a force that robot should track in specified direction \\
\hline FCPressL & To move linearly with desired force in specified direction \\
\hline FCPressC & To move circular with desired force in specified direction \\
\hline
\end{tabular}

\section{Force Control Module}

RobotStudio from $\mathrm{ABB}$ is the software environment to program the basic robot commands. RAPID codes that are created in RobotStudio can be sent to the controller unit for execution. To accomplish force control tasks, ABB force control module, being as one of libraries in the software RobotStudio, provides specific instructions for programming. Five important relevant instructions are tabulated in Table 1.1. RAPID program that commands the robot to move in a linear path and at the meantime maintain the desired force can be written in RobotStudio and it will be utilized in Chapter 4.

Besides the achievement of generating and keeping the desired polishing force, the force control is able to find out the position of workpiece surface in the specified direction. This task can be also completed by ABB force control module. The user interface of RobotStudio is shown in Fig. 1.2.5. 


\section{Chapter 2}

\section{Research Platform and Project}

\section{Objectives}

\subsection{Experimental Robotic Polishing System}

The design of a robotic polishing system is presented in this section. This system will be employed in robotic polishing experiments. Its overall configuration is composed of two main parts: an $\mathrm{ABB}$ robotic arm and a compliant polishing end-effector which will be subsequently introduced in more detail. The diagram in Fig. 2.1.1 illustrates the architecture of the proposed robotic system. As depicted in Fig. 2.1.1, the ABB 6-DOF industrial robot is maneuvered during polishing. At the same time, a cylinder-shaped polishing end-effector is designed and mounted at the end wrist of the robot. This automatic system also comprises the force sensor, the encoder sensor, the host computer and an ABB controller $\mathrm{u}-$ 


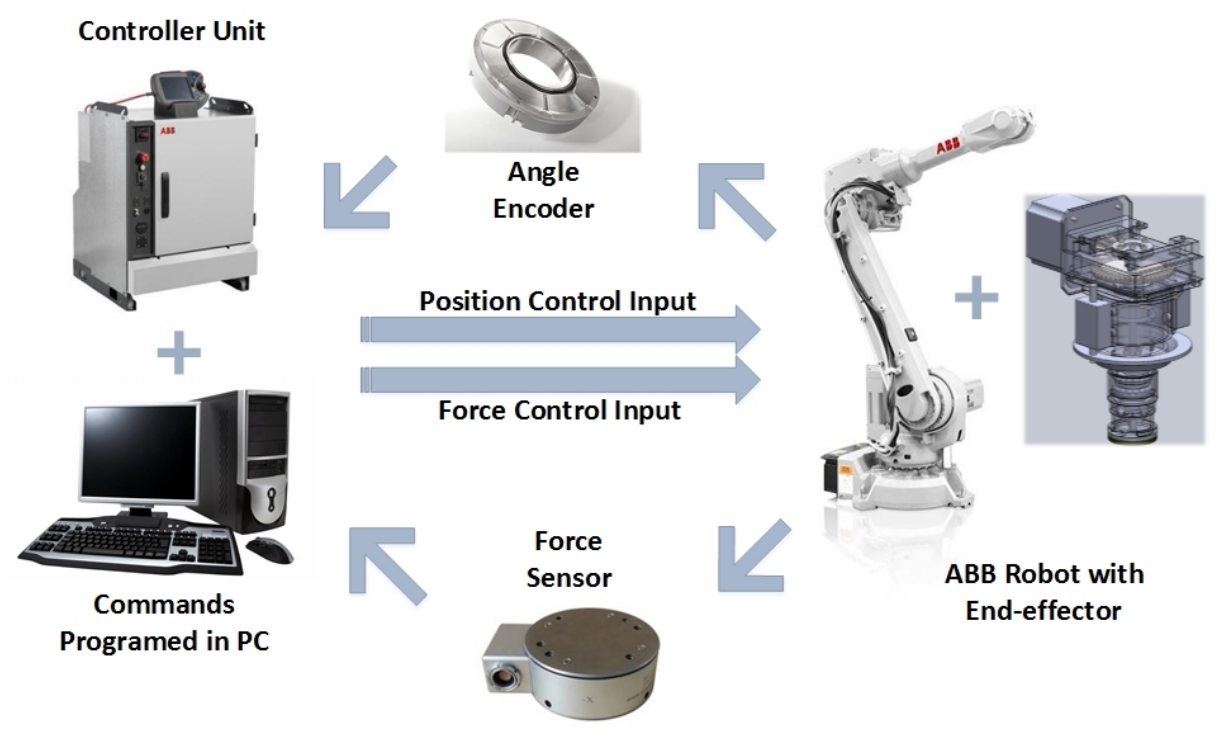

Figure 2.1.1: The closed-loop of robotic polishing system

nit. The force sensor and encoder sensor synergistically provide feedback signal to the controller unit which can generate the control input to the polishing robot. As introduced in the last chapter, the operation commands are programmed via RobotStudio in the host computer and transmitted into the controller unit.

\subsubsection{ABB Robotic Arm with End-effector}

\section{Industrial Robotic Arm}

Both CNC machine polishing and robotic polishing can somehow automate traditional polishing process. Nevertheless, the usage of industrial articulated arm enables us to enlarge the working area. Without the industrial robots, it is totally not possible to do polishing especially as workers encounter the workpiece of large size, like huge sculptures and water pipes. Added to this point, the huge 
workpieces with complicated surfaces and shapes essentially demand polishing tool to change the position and orientation from time to time. The industrial robots do have the outstanding mobility to address such workpieces. Another reason why robotic articulated arms are preferable is that they have a better flexibility than $\mathrm{CNC}$ machines.

One issue should be analyzed is the robot's position accuracy. The normal position accuracy of industrial robotic manipulator is around $100 \mu \mathrm{m}$, which may not help to control the removal of material precisely [25]. This robot's position accuracy is not sufficient especially when people request for material removal of several $\mu \mathrm{m}$ in surface position accuracy. However, this issue can be neglected given that robotic polishing in general does not need extremely precise position control which can realize the accuracy of several $\mu \mathrm{m}$. In this project, we depend on the exerted force in the tool z-direction rather than the position to achieve surface position accuracy.

The chosen manipulator model is ABB 6-DOF articulated robotic arm IRB260020 which is shown in Fig. 2.1.2. Its maximum reach is $1.65 \mathrm{~m}$ and the maximum payload is $20 \mathrm{~kg}$. In addition, the position repeatability and path repeatability are $40 \mu \mathrm{m}$ and $130 \mu \mathrm{m}$, respectively. As seen in Fig. 2.1.2, between the tip of the robotic arm and the polishing end-effector, a 6-axis force sensor is mounted and integrated with ABB robot system. This force sensor, 3HAC046093-001 is designed and produced by company Sunrise Instruments (SRI). It should be noted that the capacity of this load cell in z-direction is $165 \mathrm{~N}$. 


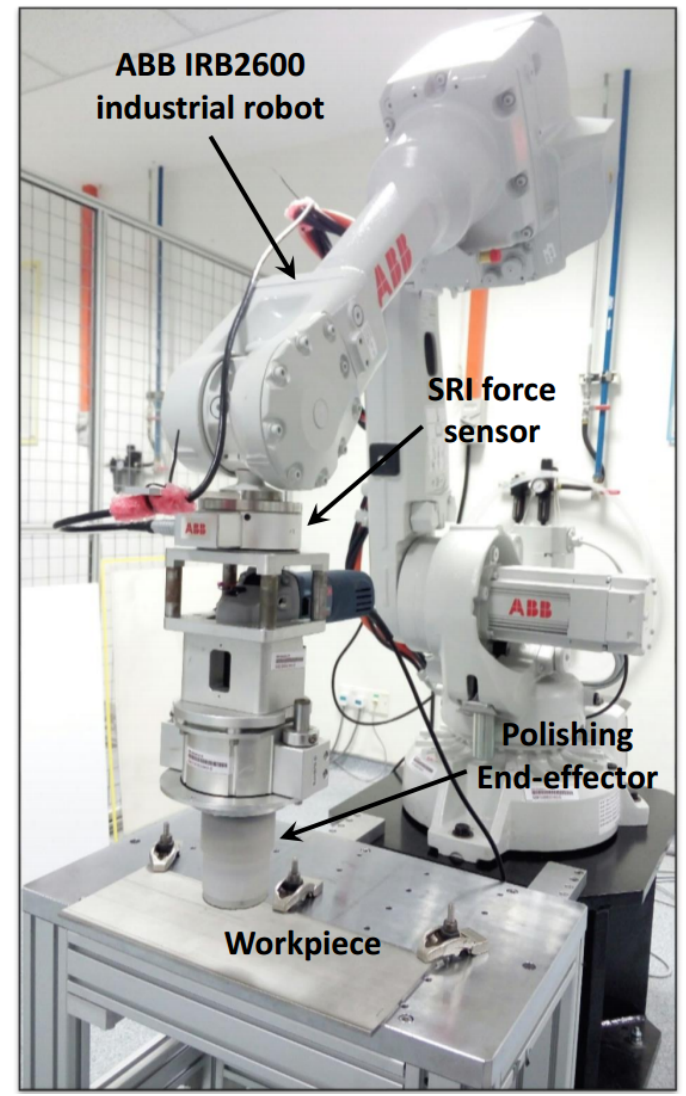

Figure 2.1.2: Experimental setup for robotic polishing

\section{Proposed Polishing End-effector}

A designed polishing end-effector is proposed in this study. Its outlook can be seen in Fig. 2.1.2 while its half-section view is revealed in Fig. 2.1.3. Rotation is the essential motion to conduct the material removal process so the proposed endeffector design contains a polishing motor (1) for rotational motion. Here, it is specified how the motion power is transmitted from the polishing motor (1) to the polishing pad (9) according to Fig. 2.1.3. As the polishing motor (1) works, one geared bevel gear turns another bevel gear that is connected to the inner spindle 


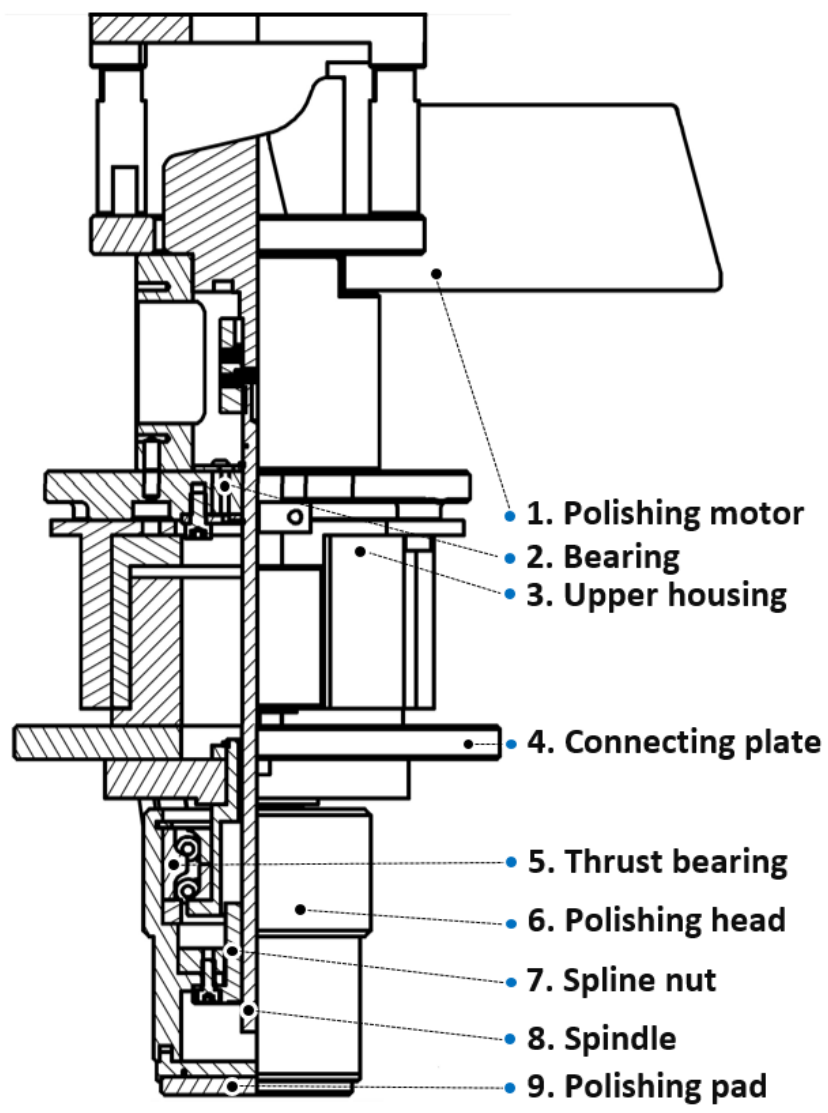

Figure 2.1.3: The sectional view of the proposed polishing end-effector

(8) and hence the spindle (8) can rotate. An important component, spline nut (7) forces the outer polishing head (6) to execute rotational motion. To realize it, this nut (7) is engaged with the spindle (8) through its raceway grooves and simultaneously connected to the cylinder polishing head (6). A circular polishing pad (9) is attached at the tip of polishing head (6) and driven by polishing head's rotation. Bearing (2) allows the relative motion between the spindle (8) and the upper housing (3). Bearing (5) is used to enable the polishing head (6) to rotate relatively to the inner stationary part which is connected to the upper housing (3) by the connecting plate (4). 
The height of this end-effector is $0.36 \mathrm{~m}$ and its total weight is $8.40 \mathrm{~kg}$. In this case, BOSCH angle grinder, GWS8-100CE, is used as polishing motor. Its main specifications are given as follows: rated power input: $850 \mathrm{~W}$; no-load speed range: 2800 r.p.m-11000 r.p.m; weight without cable: 1.8 kg. HAWK sandpapers (particle size: $100^{\#}$ grit) whose radius is $31 \mathrm{~mm}$ are selected as the polishing pad.

The proposed research platform can availably realize the robotic polishing task. Particularly, the important polishing parameters, like the polishing force, the polishing tool feed rate and the tool angular speed can be set and controlled.

\subsubsection{Polishing Performance Measurement}

To evaluate the performance of robotic polishing, in this study, two performance parameters are considered: surface material removal and surface roughness. Each measurement is carried out by one special apparatus: micrometer for surface material removal and roughness tester for surface roughness. As assessing each polished area, several measurement points that are evenly distributed are chosen. Also, for each point, we repeat its measurement for several times and take the average value.

\section{Material Removal Measurement}

In order to obtain the value of material removal, the thickness of workpiece before and after polishing process is measured and the difference between two measured results is calculated. The selected device is Mitutoyo digimatic mi- 
crometer 293-100 with $0.1 \mu \mathrm{m}$ resolution. For ensuring the equivalence, measurements before and after the polishing should be performed under the same calibration of micrometer. The usage of micrometer can be viewed in Fig. 2.1.4.

\section{Roughness Measurement}

Roughness is measured by roughness tester Mitutoyo SJ-210 with a resolution of $0.0002 \mu \mathrm{m} . \quad R a=\frac{1}{n} \sum_{i=1}^{n}\left|Y_{i}\right|$ is considered as the most common roughness parameter whose definition is the arithmetic mean of the absolute values of all evaluation profile deviations $Y_{i}$ from the mean line over the entire evaluation length ( $n$ : the total number of measurement points). How roughness tester is operated for experiments can be seen as well in Fig. 2.1.4. It should be noted that both material removal measurement and roughness measurement in this case only can be carried out in the flat surface, but not the curved surface.

\subsection{Projective Objectives}

Previous works in robotic polishing planning only cover the objective that is to achieve the maximum roughness reduction and the maximum material removal [26] [27]. The problem lies in that traditionally, they always assume that the workpiece surface is even, so their planned polishing parameters keep constant even as encountering the uneven surface. The unexpected consequence is that the under-polishing or the over-polishing occurs. In order to overcome the drawback, the projective objectives are described as follows: 

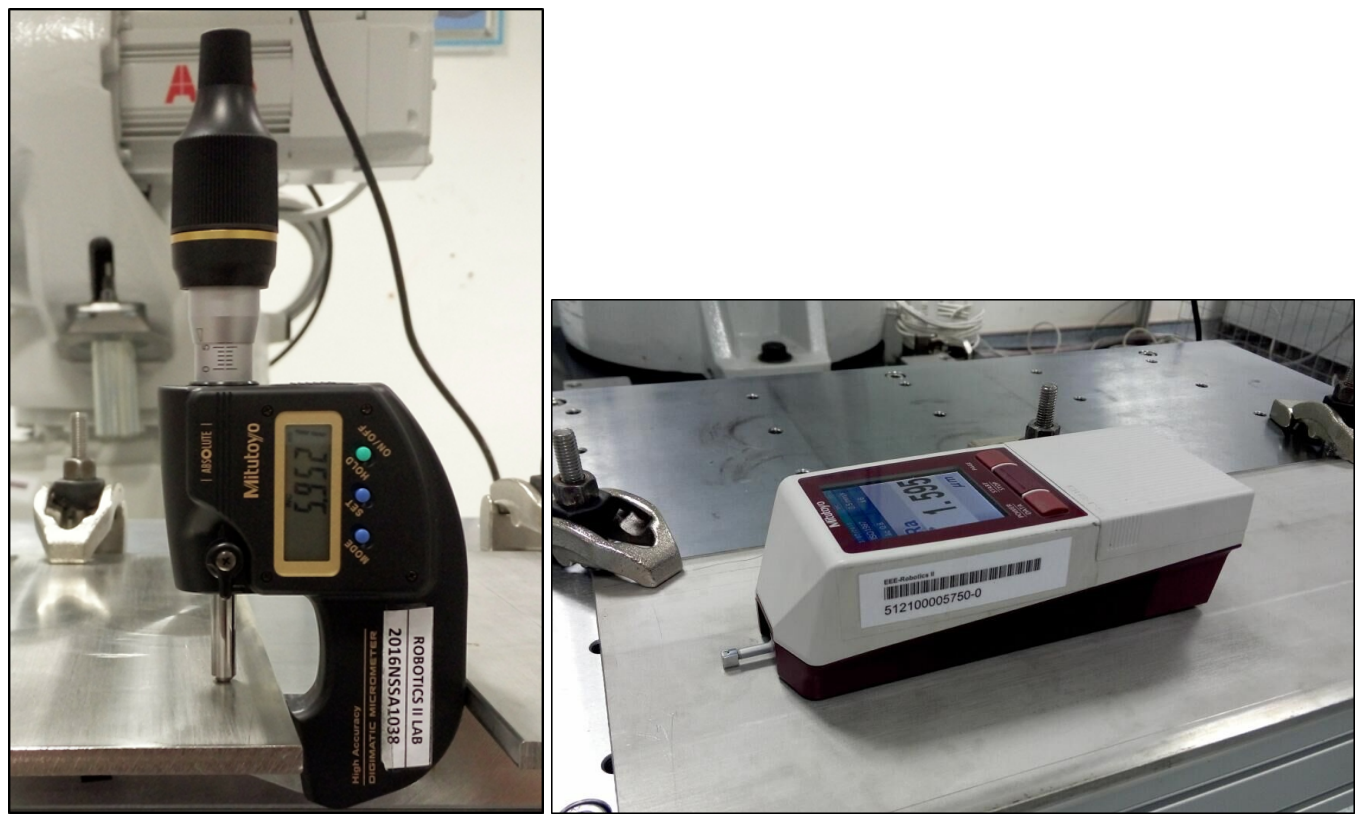

Figure 2.1.4: Material removal measurement (the left figure) and roughness measurement (the right figure) during experiments

(i) In this study, the overall planning objective becomes that the polished workpiece should have specified roughness and specified position accuracy. The most important distinction of new objective indicates that the proposed robotic polishing planning is supposed to achieve both the desired roughness reduction and the desired material removal according to uneven situations of the initial workpiece surface. The planned polishing parameters can be adjusted as the real surface is uneven and therefore the under-polishing or the over-polishing is expected to be avoided;

(ii) The first concern of the planning is related to how to accurately build up the polishing process model that can describe the relationships between polishing parameters and polishing performance. The polishing process involves with many machining parameters and physical processes and thereby the 
mechanisms of polishing manufacture are normally complicated. If the machining parameters are not properly chosen on the basis of the accurate model during polishing, the unwanted polishing performance may occur. To resolve the modelling problem in this robotic polishing planning experiment, neural network based on the collected training examples will be applied to approximate the process model;

(iii) At the same time, the second matter is to search for the optimum parameters employing the prepared model. Genetic algorithm that recursively updates the solution population should find out the best polishing parameters;

(iv) Last but not least, verification experiments will be carried out by polishing workpieces with planned polishing parameters. The ideal results should be that both the desired surface material removal and the desired surface roughness reduction are obtained. 


\section{Chapter 3}

\section{Process Planning of Uneven Surface}

\section{Polishing}

Chapter 3 introduces how the robotic polishing planning for uneven surfaces is conducted. The overall objective is to determine optimal parameters as polishing uneven surfaces and achieve ideal smoothness and flatness (surface smooth accuracy and surface position accuracy). When an uneven surface is under polishing, the material removal should be adapted to make surface totally flat. Furthermore, the roughness reduction should be controlled to obtain the same roughness over the polished surface. The particular details will be defined in the next subsection. Modeling of the polishing process helps us to further understand industrial polishing mechanisms and then choose optimal polishing parameters. However, it is pretty difficult to figure out process characteristics. To overcome modelling problem, neural network (NNW) is employed to approximate the pol- 
ishing process. Then, search of polishing parameter has been accurately done and specifically genetic algorithm (GA) is utilized.

Traditional mechanism models based on repetitive empirical experiments that are certainly time-consuming and physical modelling that are related to quite a few parameters are not accurate enough [28]. They are also difficult to apply as some associated parameters are not easy to obtain and the required machining conditions are normally of a limited range. Therefore, for process characteristic formulation, NNW, a powerful computational intelligence technique, comes up which has an excellent capacity to approximate unknown process and make predictions. Benardos [28] and Markopoulos [29] have employed NNW to predict the surface roughness in milling process. Besides applications in manufacture process formulation, $\mathrm{NNW}$ is widely used in other fields, like image processing, automated control system, financial prediction, etc [30] [31].

GA was raised for the first time by Holland in 1975 [32]. It is usually viewed as an effective stochastic search technique based on the evolutionary principle. The GA structure aims to evolve the chromosome population that represents a set of the basic random solutions through genetic operators: selection, crossover and mutation. This process keeps working until the individual with satisfying fitness value that is equal to the optimal solution appears. GA technique has been proved to be highly accurate in planning problems [33] [34]. In [35], Hou et. al apply GA to find optimal parameters for a nano-particle milling process. In addition to its accurate performance, GA is known for its excellent robustness [36] [37]. 
Synergistic work of NNW and GA draws much attention in process planning problem for surface technology [38] [39]. Shen et. al [40] presented a combined NNW and GA method to optimize the injection molding process. NNW integrated with GA has been used to predict minimum surface roughness by scholars in [26]. The process optimization based on NNW and GA for a polishing tool to achieve both minimum roughness and high efficiency of roughness improvement is studied in [27].

\subsection{Robotic Polishing Task for Uneven Surfaces}

A robotic polishing task is particularly defined. As depicted in Fig .3.1.1, the unpolished workpiece has a generally flat surface. But the surface is not totally even because the $1^{\text {st }}$ surface and the $2^{\text {nd }}$ surface have different values in required material removal $\left(M R_{1}\right.$ and $\left.M R_{2}\right)$ and required roughness improvement $\left(\Delta R a_{1}=R a_{f}-R a_{1}\right.$ and $\left.\Delta R a_{2}=R a_{f}-R a_{2}\right)$. All in all, the planning purpose is to automatically determine two different groups of polishing parameters to eliminate the thickness difference as well as the roughness difference between two surfaces. The expected consequence should be that the uneven surface becomes an even one with the same height and the same roughness $R a_{f}$ after polishing. The robotic polishing system introduced in Chapter 2 is operated to perform the task. The robotic manipulator is programmed to carry the polishing end-effector to slide along the workpiece for only single trip. 


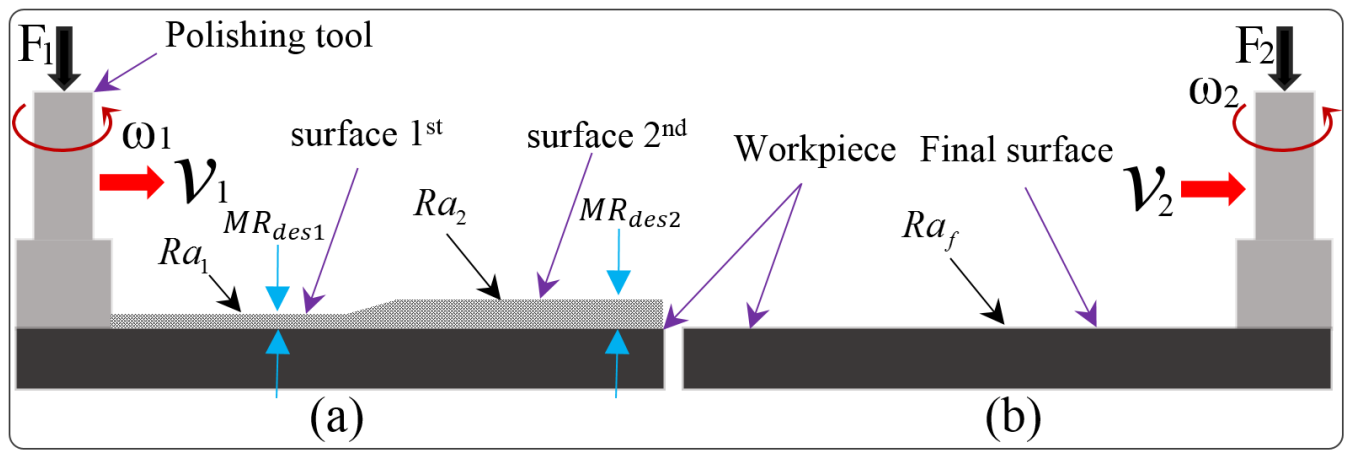

Figure 3.1.1: Uneven surface polishing (a) Unpolished surface with two different heights/roughness (b) Polished surface with the identical height/roughness

Currently, polishing uneven surfaces to achieve surface smooth accuracy and surface position accuracy is definitely needed. In engine blade repairing application, the welding process is involved, which may generate excess welded portions. Even the small differences between welded area and unwelded area can result in the deterioration of engine performance [41]. Therefore, it is usually required that welded surfaces should be milled and polished to be almost equal to unwelded surfaces.

\subsection{Polishing Planning Procedure}

The first step of the proposed solution is that NNW is used to establish a model between controllable polishing parameters and polishing performance. Polishing process is complicated to formulate because it involves many physical actions, like cutting, rubbing and ploughing. The usage of NNW is able to learn complicated relationships inside the polishing process. For the second step, each 
Table 3.1: Polishing parameters and polishing performance

\begin{tabular}{c|c|c|c|c}
\hline \multicolumn{3}{c|}{ Polishing parameters } & \multicolumn{2}{c}{ Polishing performance } \\
\hline Polishing & Rotational & Feed & Material & Roughness \\
force & speed & rate & removal & improvement \\
$(\mathrm{N})$ & $($ Level $)$ & $(\mathrm{mm} / \mathrm{s})$ & $(\mathrm{mm})$ & $(\mu \mathrm{m})$ \\
\hline
\end{tabular}

machining parameter is planned by GA according to machining requirements of uneven surface polishing task. The proposed algorithm is demonstrated in Fig. 3.2.1 and its procedures are summarized as follows:

(i) A number of experiments are conducted to collect training data set. From Table 3.1, polishing force, tool rotation speed and tool feed rate are chosen as polishing conditions while the polishing performance are evaluated by material removal and roughness improvement. Collected data samples are given to train NNW. NNW finally builds up a relationship between polishing conditions and polishing performance. In addition to NNW training process, the testing process cannot be ignored to see if or not the trained NNW model is correct.

(ii) Trained NNW model, the desired material removal and the desired roughness improvement are together provided for GA and then GA is responsible to search for the optimal polishing parameters;

(iii) The optimal polishing parameters that are generated by GA are finally applied in the polishing process. The actual polishing performance using planned parameters should be compared to the desired polishing performance to evaluate whether the proposed strategy is effective or not. 


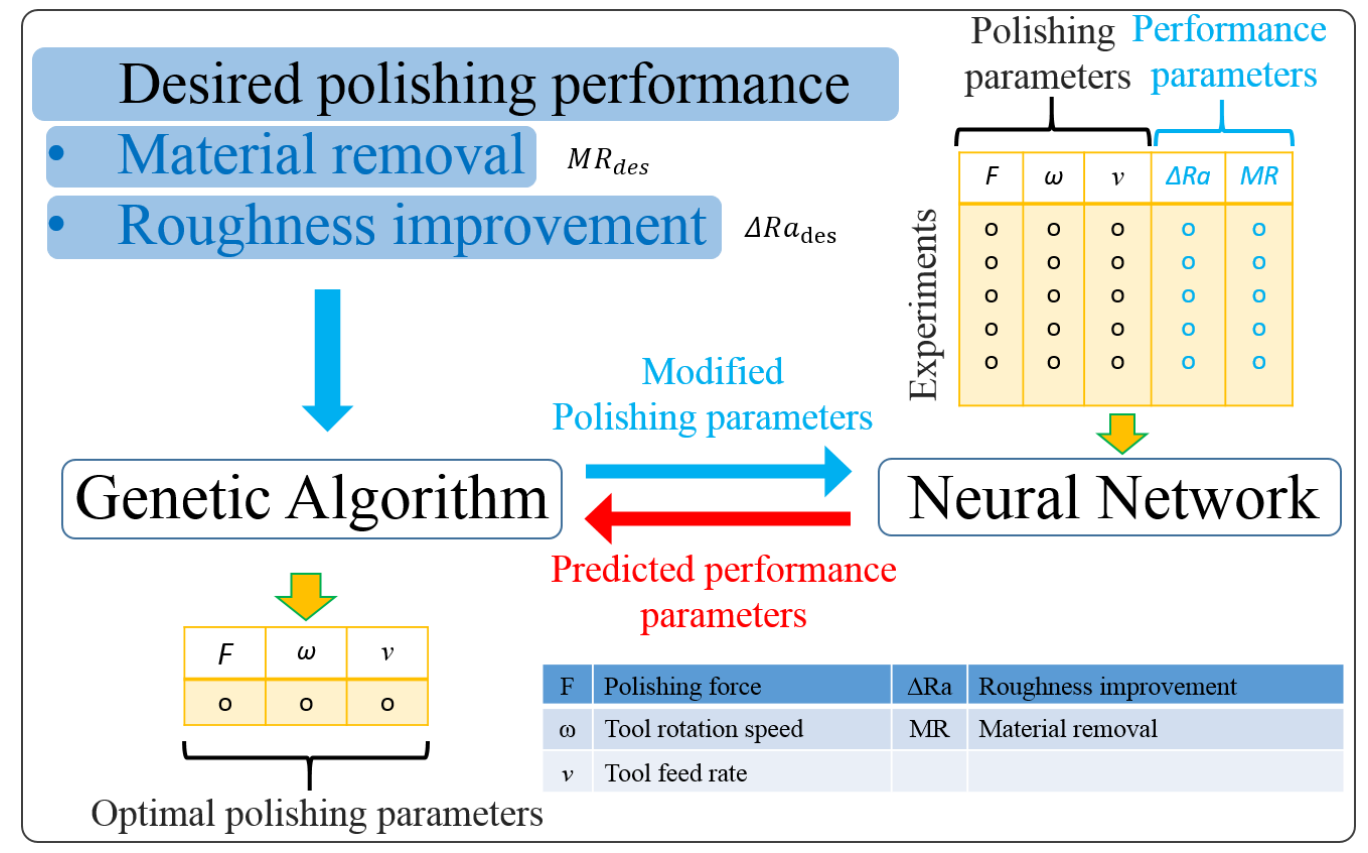

Figure 3.2.1: The proposed planning algorithm for polishing uneven surface

\subsubsection{Neural Network Approximation}

\section{Network Design}

NNW should be well specified before training based on the polishing process. From Fig. 3.1.1, $F_{z}$ : the polishing force in tool z-direction, $\omega$ : rotational speed of the end-effector and $v_{f}$ : feed rate of the end-effector along the surface are considered as polishing parameters. The polishing performance are basically assessed in surface smooth accuracy and surface position accuracy. We define roughness improvement $\triangle R a=R a_{\text {initial }}-R a_{\text {final }}$ for smooth accuracy and material removal $M R$ for position accuracy, respectively.

The network architecture with one input layer, one output layer and hidden layers is depicted in Fig. 3.2.2. In terms of the previous analysis, $F_{z}, \omega$ and 


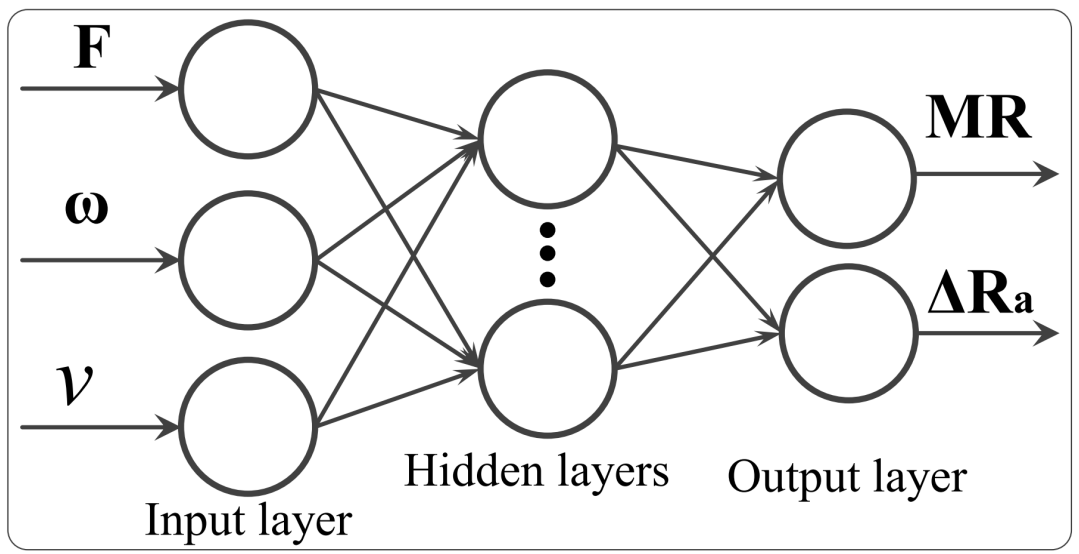

Figure 3.2.2: NNW architecture

$v_{f}$, these three parameters are chosen as NNW training inputs. Accordingly, the roughness improvement $\triangle R a$ and material removal $M R$ are selected as NNW training target outputs. The feedforward network structure basically consists of a number of neurons and their connections. The connection between two nodes has its corresponding weight and the intrinsic principle of NNW training is to update the value of every weight. To achieve good approximation, the number of hidden layers and the number of nodes in each hidden layer should be appropriately chosen.

\section{Training Process}

The experimental training samples enable NNW to be trained again and again and then function as an available polishing model. Training data is fed into the input layer. The hidden layers process the data and the NNW output is generated through the output layer. Every neuron of the network in Fig. 3.2.2 can be seen as a calculator with an nonlinear activation function. For every node, the 
linear combination of multiplication of each input by its respective weight will be processed by the activation function to feed out the node's output.

NNW is iteratively adjusting its weights by repetitive training according to the designed adaption law. Here, the conventional backpropagation law is employed for this recurrent process which considers the error function, sum of squared errors between actual NNW outputs and sample target outputs, as objective function. To be more specific, the Levenberg-Marquardt formula, one of backpropagation algorithms has been applied in this study and more details will be later introduced [42]. The training goal is to look for the weights which can minimize the objective function as far as possible.

\subsubsection{Genetic Algorithm Planning Procedure}

The defined polishing task is to get a extremely flat surface with homogeneous roughness. Given that the polishing model that is represented by NNW is completely known and predictable, GA strives to search for the optimal polishing parameters to accomplish this polishing task. The procedures of GA are summarized in Fig. 3.2.3 where the whole process is divided into three main parts: initialization, fitness value estimation and evolution operator.

\section{Initialization}

As shown in the flowchart from Fig. 3.2.3, the first step of GA is to create a random initial population of a certain size and encode each member (chromo- 
some/individual/candidate) of population. Each member among the population represents a possible solution. The ultimate goal of GA is to find out the optimum chromosome.

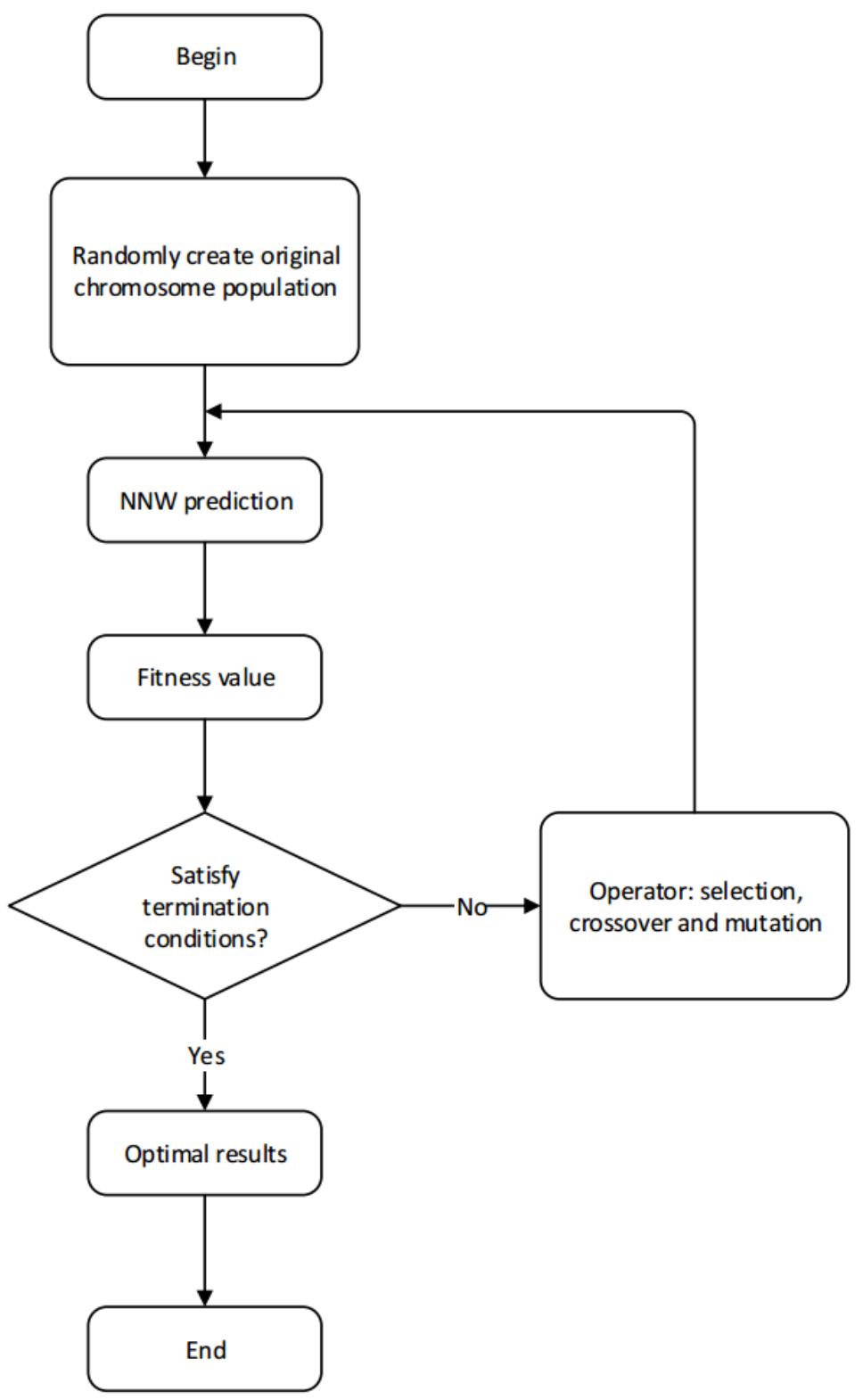

Figure 3.2.3: GA planning process with NNW prediction 


\section{Fitness Value Estimation}

The stopping criterion determines whether or not GA is supposed to stop. How to make this decision depends on the the current colony's fitness value computed by designed fitness function. Each individual's fitness should be calculated and evaluated. The fitness value implicitly represents the quality of the individual. Thereby, the devised fitness function significantly influences the overall search direction. The searching process of GA iterates until the fitness value reaches the stopping criterion.

\section{Evolution Operator}

If the termination condition was not satisfied, GA renews the individuals in the current generation to create the next generation. To form the next generation, the evolution operator helps to update the chromosomes via selection operation, crossover operation and mutation operation. The selection operation is working to choose a certain percentage of chromosomes who have better fitness value as parents. The genes from selected parents are then contributed to create children in the crossover operation. Each offspring borrows the features from both parents. A small proportion of single parents are selected to make random changes in their genes and thereby create mutation children. The mutation operation is responsible for leading to genetic diversity and makes GA to search in a boarder space.

Besides crossover children and mutation children, the new generation con- 
tains elite children. Parts of chromosomes whose fitness values are outstanding in the current generation are passed directly to the next generation and they are called elite children.

Evolution operator which generates new solutions is the principal component of genetic algorithm. Its exploration bases on the selection of successful parents and the elimination of the weak parents. It also allows the greater diversity of possible solutions because of the random nature of the mutation operation. The parallel work of three operations makes the algorithm converge towards highquality solutions. 


\section{Chapter 4}

\section{Experimental Results}

Experimental studies are introduced in this chapter. The employed polishing robotic setup is revealed in Fig. 2.1.2. Several cuboid blocks of workpieces with $160 \mathrm{~mm}$ length, $60 \mathrm{~mm}$ width and $6 \mathrm{~mm}$ height are selected for experiments. Their material is stainless steel SUS304. As shown in Fig. 4.0.1, the material removal and the roughness are obtained by calculating the average of 5 positions that are evenly distributed in one area that covers a distance of $40 \mathrm{~mm}$.

\subsection{Neural Network Training}

Several NNW training samples which are particularly tabulated in Table 4.1 are collected to train NNW for the first procedure. Each sample contains polishing conditions and corresponding polishing performances. On the one hand, the training input variables are the polishing force in the tool z-direction $F_{z}$, tool 


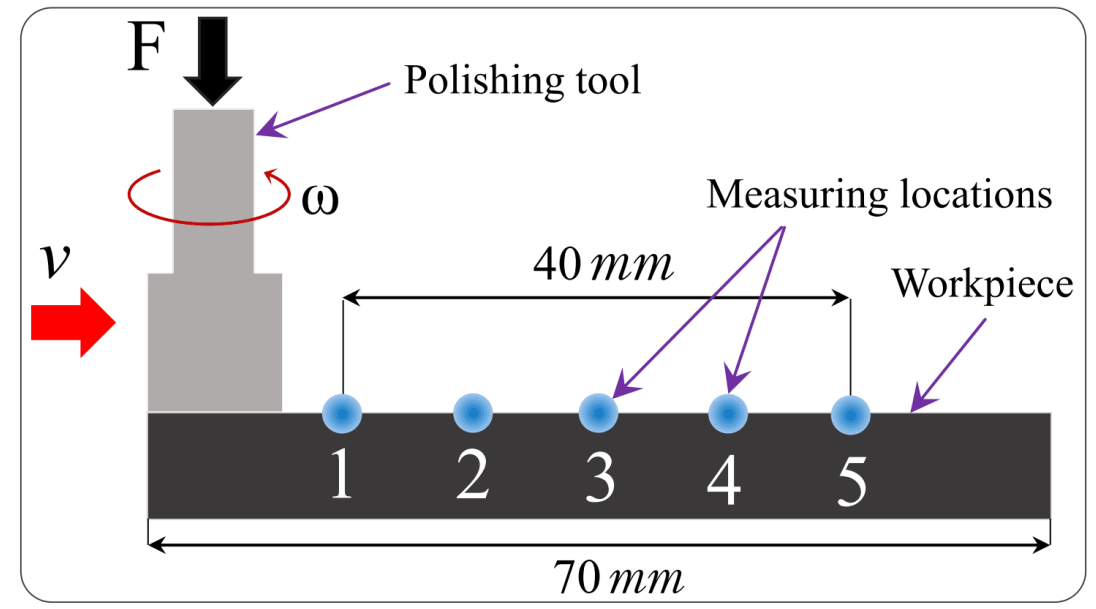

Figure 4.0.1: The illustration of measurement sampling

rotational speed $\omega$ and tool feed rate in the tangential direction $v_{f}$. On the other hand, the training target outputs consist of material removal $M R$ and roughness improvement $\triangle R a$.

It is set up for NNW training that $F_{z}$ has 9 levels, $\omega$ has 6 levels and $v_{f}$ has 4 levels. Considering that to execute experiment is tedious and time-consuming, Only 25 groups are selected for experiments rather than 216 groups. Yet, the polishing parameters in 25 groups are not determined randomly. Specifically, Taguchi design method is applied to have most effective multi-factor combination [35].

Matlab software is then employed to create and train the NNW that has one hidden layer with exactly 10 neurons. More specific information of NNW training process is presented as follows:

(i) Both input and output of training data are normalized to a specific range $[0.1,0.9]$ before the training. Normalization enables training to be faster and 
reduce the possibility of getting stuck in local optima. Although training without normalization is allowed, the trained NNW might be not effective due to that magnitudes of different features differ a lot. The normalization function is given as follows

$$
\bar{x}=0.1+0.8 \frac{x-x_{\min }}{x_{\max }-x_{\min }}
$$

where the sample data $x$ is normalized into $\bar{x} ; x_{\max }$ and $x_{\min }$ are the maximum and minimum value for $x$, respectively.

(ii) The activation function whose purpose is to introduce nonlinear relationships is defined by the following expression

$$
s(\mathbf{u})=\frac{2}{1+e^{-2 \mathbf{u}}}-1
$$

where $\mathbf{u}$ is the input vector for one neuron and $s(\mathbf{u})$ is the according output vector. This nonlinear function is so-called hyperbolic tangent sigmoid transfer function.

(iii) The weights are being updated recursively by Levenberg-Marquardt algorithm which is already proven to provide fast and accurate training for $\mathrm{N}$ NW [43]. The expression is given as follows:

$$
\mathbf{W}_{k+1}=\mathbf{W}_{k}-\left(\mathbf{J}^{T} \mathbf{J}+\mu \mathbf{I}\right)^{-1} \mathbf{J}^{T} \mathbf{e}
$$


where $\mathbf{W}_{k+1}$ and $\mathbf{W}_{k}$ are the weight vector before and after one adaption, respectively. Their initial values are arbitrarily chosen to be $\mathbf{0}$ and fortunately, such settings are examined by the NNW training performance and the NNW testing performance. $\mathbf{J}$ is the Jacobian matrix that includes first derivatives of the error vector e with respect to the weights. The learning of the weights, $\mu$, is a changeable scalar whose initial value is $0.001 . \mu$ will be increased by 10 until the error $e$ becomes less than the setting goal. Once the error $e$ keeps being less than the setting goal, $\mu$ starts to decrease by 0.1 for each epoch.

(iv) The training performance is assessed by mean squared error. The training will stop once the mean squared error eventually is less than the default goal value or the training epochs reaches 1000. How the mean squared error varies is shown in Fig. 4.1.1.

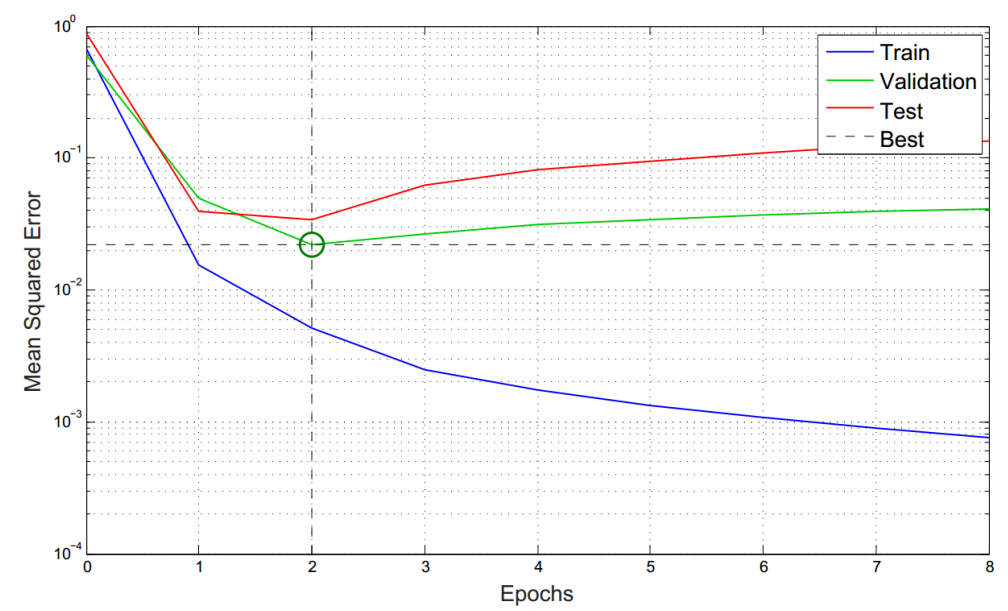

Figure 4.1.1: The mean square error for the training epochs 
Table 4.1: Experimental results of NNW training samples

\begin{tabular}{c|c|c|c|c|c}
\hline No. & $\begin{array}{c}\text { Polishing force } \\
(\mathrm{N})\end{array}$ & $\begin{array}{c}\text { Rotational speed } \\
(\text { Level })\end{array}$ & $\begin{array}{c}\text { Feed rate } \\
(\mathrm{mm} / \mathrm{s})\end{array}$ & $\begin{array}{c}\text { MR } \\
(\mathrm{mm})\end{array}$ & $\begin{array}{c}\triangle \mathrm{Ra} \\
(\mu \mathrm{m})\end{array}$ \\
\hline 1 & 5 & 2 & 80 & 0.0026 & 0.115 \\
2 & 5 & 3 & 20 & 0.0018 & 0.519 \\
3 & 5 & 5 & 40 & 0.0012 & 0.373 \\
4 & 10 & 1 & 80 & 0.0022 & 0.585 \\
5 & 10 & 4 & 20 & 0.0027 & 0.739 \\
6 & 10 & 6 & 40 & 0.0025 & 0.696 \\
7 & 15 & 2 & 40 & 0.0013 & 0.661 \\
8 & 15 & 4 & 60 & 0.0025 & 0.768 \\
9 & 15 & 6 & 80 & 0.0030 & 0.617 \\
10 & 20 & 1 & 60 & 0.0010 & 0.484 \\
11 & 20 & 3 & 40 & 0.0037 & 1.256 \\
12 & 25 & 1 & 20 & 0.0029 & 0.578 \\
13 & 25 & 4 & 40 & 0.0028 & 1.153 \\
14 & 25 & 5 & 60 & 0.0051 & 0.808 \\
15 & 30 & 1 & 40 & 0.0030 & 0.544 \\
16 & 30 & 5 & 80 & 0.0044 & 0.613 \\
17 & 35 & 3 & 80 & 0.0049 & 0.916 \\
18 & 35 & 5 & 20 & 0.0026 & 1.144 \\
19 & 35 & 6 & 60 & 0.0066 & 0.838 \\
20 & 40 & 2 & 20 & 0.0057 & 1.153 \\
21 & 40 & 3 & 60 & 0.0035 & 1.211 \\
22 & 40 & 4 & 80 & 0.0039 & 0.894 \\
23 & 45 & 1 & 40 & 0.0038 & 0.999 \\
24 & 45 & 2 & 0.0035 & 0.919 \\
25 & 45 & 5 & 0.0039 & 1.202 \\
\hline
\end{tabular}




\subsection{Neural Network Testing}

In this experiment, several groups of testing samples are obtained. Here, 5 groups of parameters are randomly chosen, and their corresponding experimental results are given to check the accuracy of the trained NNW model. The testing samples are not allowed to be used in the NNW training stage.

The comparison results are set out in Table 4.2. From Table 4.2, the predictions that calculated by the NNW saved previously are almost consistent with the actual experimental values even though the relative errors exist. The average relative errors for material removal and roughness improvement are $8.4 \%$ and $15.0 \%$ respectively, which turn out to be normal compared to the previous works. Hence, it can be concluded the trained NNW model can basically match the practical polishing mechanism.

It has to be stressed that the output of NNW should be processed by denormalization which is an inverse calculation of normalization. The de-normalization equation is given as follows:

$$
y=y_{\min }+\frac{(\bar{y}-0.1)\left(y_{\max }-y_{\min }\right)}{0.8}
$$

where $\bar{y}$ is de-normalized into $y ; y_{\max }$ and $y_{\min }$ are the maximum and minimum value for $y$, respectively. 
Table 4.2: Experimental results of NNW testing samples

\begin{tabular}{c|c|c|c|c|c|c|c}
\hline No. & $\begin{array}{c}\text { Polishing } \\
\text { force } \\
(\mathrm{N})\end{array}$ & $\begin{array}{c}\text { Rotational } \\
\text { speed } \\
(\text { Level })\end{array}$ & $\begin{array}{c}\text { Feed } \\
\text { rate } \\
(\mathrm{mm} / \mathrm{s})\end{array}$ & $\begin{array}{c}\mathrm{MR} \\
(\mathrm{NNW}) \\
(\mathrm{mm})\end{array}$ & $\begin{array}{c}\text { MR } \\
(\text { actual }) \\
(\mathrm{mm})\end{array}$ & $\begin{array}{c}\triangle \mathrm{Ra} \\
(\mathrm{NNW}) \\
(\mu \mathrm{m})\end{array}$ & $\begin{array}{c}\triangle \mathrm{Ra} \\
(\text { actual }) \\
(\mu \mathrm{m})\end{array}$ \\
\hline 1 & 10 & 5 & 40 & 0.0022 & 0.0023 & 0.629 & 0.665 \\
2 & 20 & 4 & 60 & 0.0039 & 0.0032 & 0.626 & 0.821 \\
3 & 20 & 2 & 60 & 0.0023 & 0.0022 & 0.767 & 0.698 \\
4 & 25 & 1 & 30 & 0.0032 & 0.0028 & 0.755 & 0.646 \\
5 & 45 & 4 & 80 & 0.0038 & 0.0039 & 0.927 & 1.061 \\
\hline
\end{tabular}

\subsection{Genetic Algorithm Optimization}

It is critical to properly select the value of machining parameters during polishing. The effects of these controllable parameters should be firstly understood. The polishing force and rotational rate cannot be too low otherwise the material removal would be small. Also, low tool rotational speed increases the number of fine polishing lines, which influences the roughness improvement. However, that does not mean the force and rotational rate can be extremely high. In addition to mechanical damages like over-scraping, too much pressure and high rotational rate may generate a lot of heat. That has negative impacts on the roughness improvement since affected zones over the surface is easy to form under the high temperature [5]. The slower feed rate indicates more polishing time and thus more material removal/more roughness reduction, but it surely decreases the polishing efficiency [44] [45].

To determine the certain value of each parameter, GA is used to fulfill the 
searching work and its specific procedures are described as follows:

(i) The first step for GA optimization is to randomly generate an initial population of chromosomes that account for polishing parameter solutions. Each chromosome has three variables in the interval of $[0.1,0.9]$;

(ii) Then we employ the saved NNW network to predict corresponding $M R$ and $\triangle R a$ for every chromosome candidate that belongs to the current population;

(iii) The tertiary procedure is to calculate fitness value of each chromosome in the current generation. The optimization goal to simultaneously minimize two errors yields the fitness function as follows:

$$
G=\alpha_{1}\left(M R_{\text {des }}-M R\right)^{2}+\alpha_{2}\left(\triangle R a_{\text {des }}-\triangle R a\right)^{2}
$$

where $M R_{\text {des }}$ and $\triangle R a_{\text {des }}$ are the desired surface material removal and desired surface roughness improvement. $M R$ and $\triangle R a$ are predicted material removal and roughness improvement obtained from NNW. $\alpha_{1}$ and $\alpha_{2}$ are positive coefficients for material removal and roughness, respectively. This fitness function aims to search for the polishing parameters which can achieve the desired polishing performance in material removal and roughness improvement. It can be observed that better solution candidates certainly have smaller fitness value. As long as the designed termination condition is satisfied, GA comes to an end; 
(iv) Supposed that the termination condition is not met yet, the process to evolve the chromosomes through selection operation, crossover operation and mutation operation should be conducted until the satisfying fitness value appears or the iteration times reaches to a desired number. To execute selection operation, Matlab option Stochastic uniform is chosen. The way two parents perform crossover to create a new offspring is specified by option Scattered. In the new generation, the certain percents of population are children that generated from crossover operation. Lastly, mutation operation is determined by the option Gaussian;

Fig. 4.3.1 illustrates the variation of colony fitness values during GA planning process for one surface area. The best individual constantly has a relatively small fitness value over 30 generations. Nevertheless, the mean fitness value experiences some fluctuations before 11 iterations. It then starts to decrease after 21 iterations and eventually converges to a very small level. This variation demonstrates the integration of the fitness value function and evolution operator is really optimizing solution population regarding desired requirements;

(v) Once GA algorithm halts, the optimal polishing parameters can be provided. It is noted that the optimal chromosomes should be de-normalized (4.4) taking into account both the minimum and maximum limitation. The ac- 


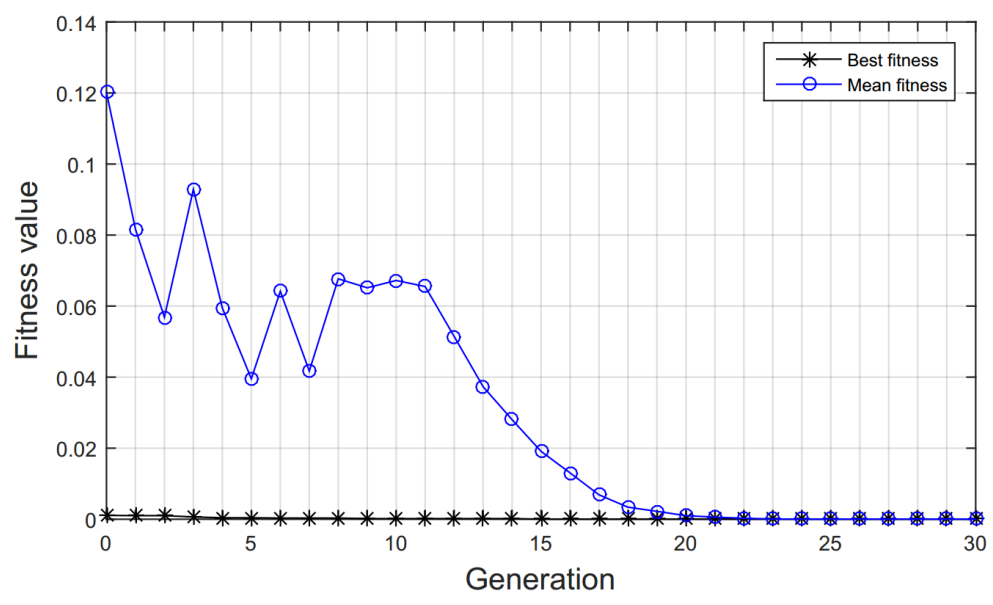

Figure 4.3.1: Variation of GA fitness value

cording limitations for three variables are expressed as follows:

$$
\begin{aligned}
& 5 \leq F_{z} \leq 45, \\
& 1 \leq \omega \leq 6 \\
& 20 \leq v_{f} \leq 80 ;
\end{aligned}
$$

additionally, relevant parameters for GA are specified: $\alpha_{1}=1 ; \alpha_{2}=1$; population size: 300; maximum iteration times: 30; mutation probability: 0.01; crossover fraction: 0.5 ; elite fraction: 0.05 .

\subsection{Planned Parameters and Verification Results}

Two workpieces are chosen to carry out the verification experiment. For the $1^{\text {st }}$ surface, the desired material removal is $M R_{\text {des }}=0.0020 \mathrm{~mm}$. At the same time, $M R_{\text {des }}$ is set to $0.0050 \mathrm{~mm}$ for the $2^{\text {nd }}$ surface. In addition, the desired 
roughness improvement $\triangle R a_{\text {des }}$ is required to be $0.700 \mu \mathrm{m}$ for both two blocks. The optimal polishing parameters that are calculated by GA procedures are identified as follows:

(i) $1^{\text {st }}$ Surface: $18 \mathrm{~N}\left(F_{z}\right)$, Level $2(\omega), 50 \mathrm{~mm} / \mathrm{s}\left(v_{f}\right)$;

(ii) $2^{\text {nd }}$ Surface: $24.5 \mathrm{~N}\left(F_{z}\right)$, Level $5(\omega), 60 \mathrm{~mm} / \mathrm{s}\left(v_{f}\right)$.

To illustrate the effectiveness of the proposed algorithm for polishing an uneven surface, the optimization experimental results are presented in Fig. 4.4.1, 4.4.2 and 4.4.3. As depicted in Fig. 4.4.3, the actual average material removal $M R\left(0.0021 \mathrm{~mm}\right.$ for the $1^{\text {st }}$ surface and $0.0048 \mathrm{~mm}$ for the $2^{\text {nd }}$ surface $)$ is nearly close to the desired specification $M R_{\text {des }}\left(0.0020 \mathrm{~mm}\right.$ for the $1^{\text {st }}$ surface and $0.0050 \mathrm{~mm}$ for the $2^{\text {nd }}$ surface).

Moreover, the good matching between the real roughness improvement $\triangle R a$ $\left(0.813 \mu \mathrm{m}\right.$ for the $1^{\text {st }}$ surface and $0.696 \mu \mathrm{m}$ for the $2^{\text {nd }}$ surface) and the desired roughness improvement $\triangle R a_{\text {des }}\left(0.700 \mu \mathrm{m}\right.$ for the $1^{\text {st }}$ surface and $0.700 \mu \mathrm{m}$ for the $2^{\text {nd }}$ surface) is also achieved. Thereby, we summarise from the verification results that the proposed algorithm is eligible to accomplish the task for polishing uneven surfaces. The desired surface material removal and the desired surface roughness reduction can be both achieved by using the planned polishing parameters. Except for these two groups of planning results with verifications, some other planning results without verifications can be checked in Table 4.3. 


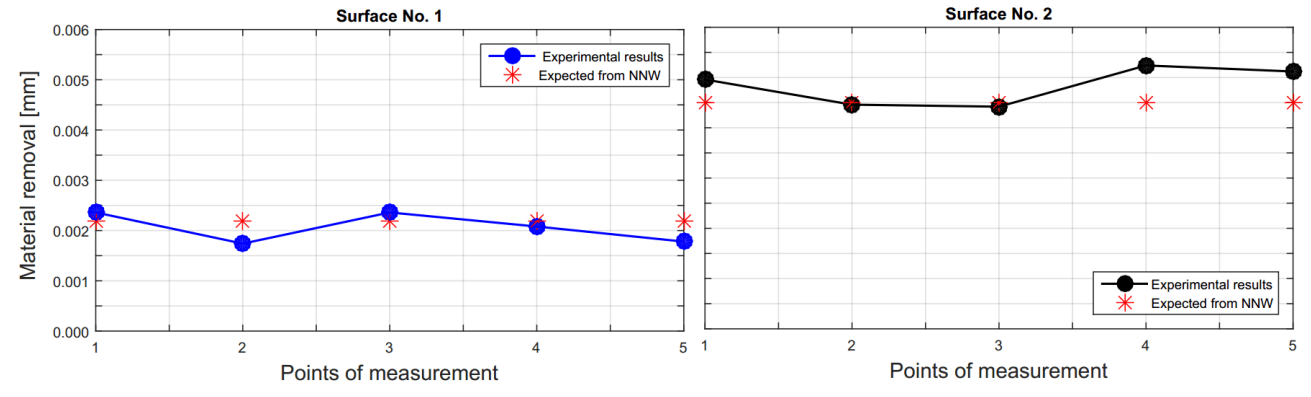

Figure 4.4.1: The experimental results for the $1^{\text {st }}$ surface and the $2^{\text {nd }}$ surface in material removal
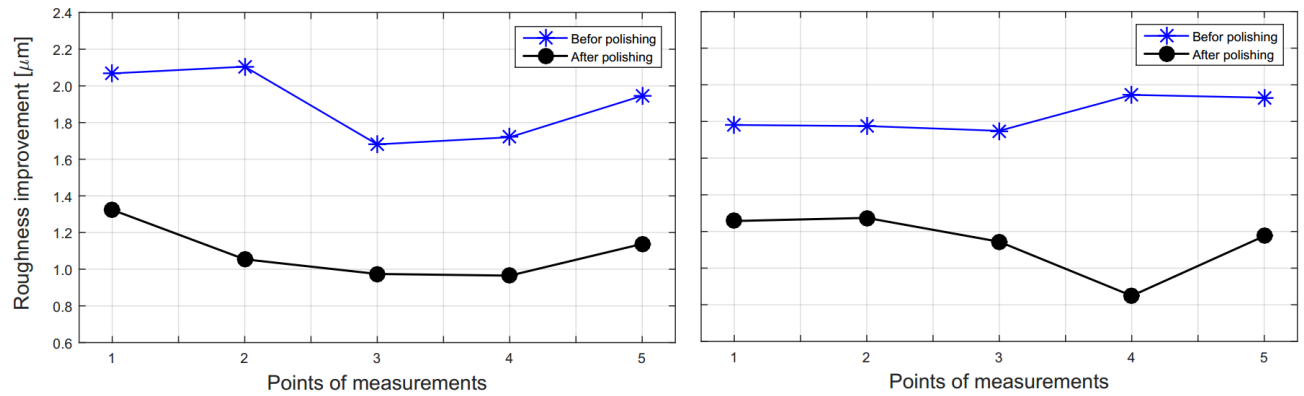

Figure 4.4.2: The experimental results for the $1^{\text {st }}$ surface and the $2^{\text {nd }}$ surface in roughness improvement 

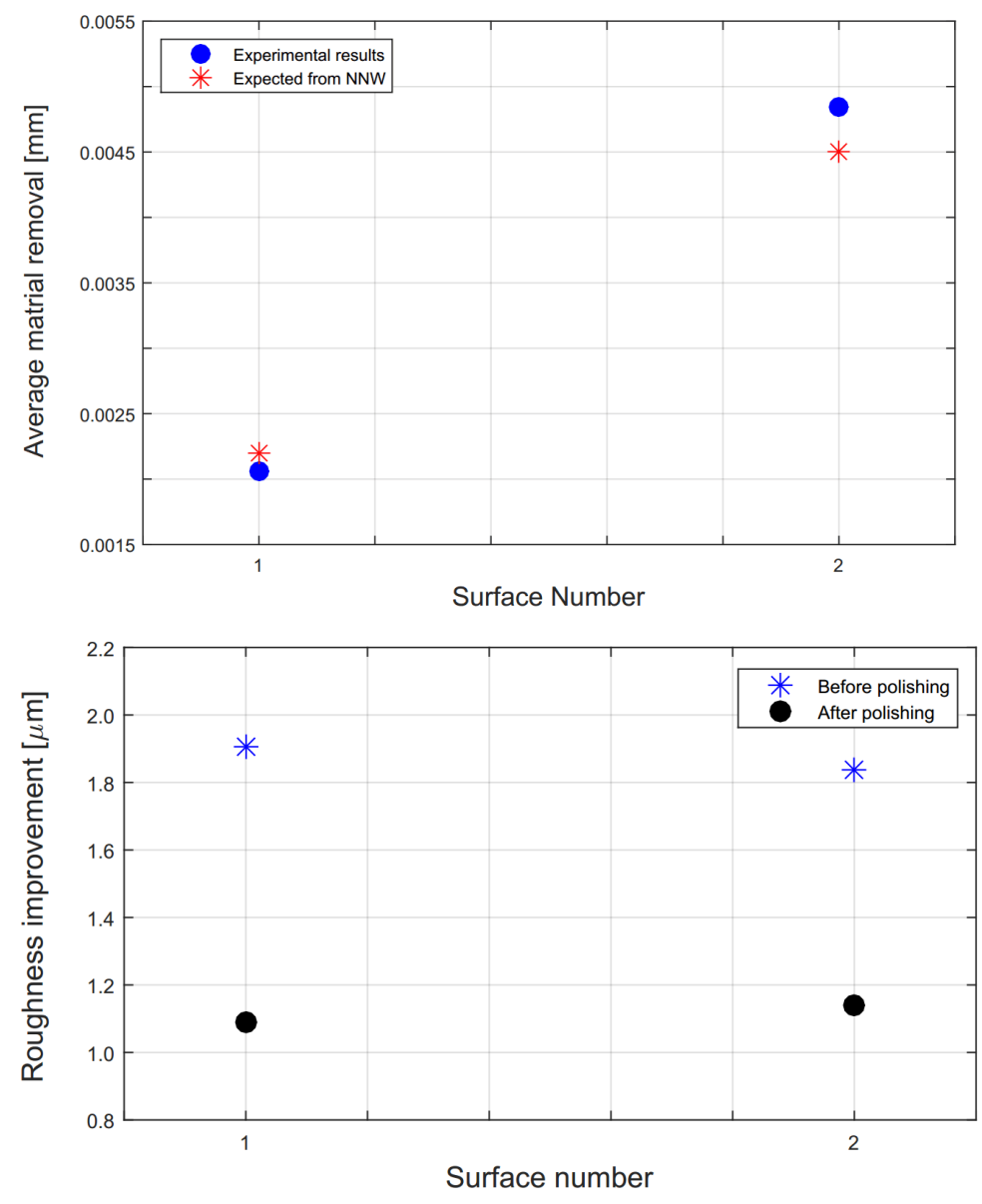

Figure 4.4.3: The average material removal/roughness improvement for the $1^{\text {st }}$ surface and the $2^{\text {nd }}$ surface 
Table 4.3: Planned results with desired performance

\begin{tabular}{c|c|c|c|c}
\hline \multicolumn{2}{c|}{ Desired performance } & \multicolumn{3}{|c}{ Planned polishing parameters } \\
\hline$M R_{d e s}$ & $\triangle R a_{d e s}$ & $F_{z}$ & $\omega$ & $v_{f}$ \\
$(\mathrm{~mm})$ & $(\mu \mathrm{m})$ & $(\mathrm{N})$ & $($ Level $)$ & $(\mathrm{mm} / \mathrm{s})$ \\
\hline 0.0010 & 0.700 & 13.5 & 2 & 40 \\
0.0030 & 0.700 & 27.5 & 3 & 65 \\
0.0040 & 0.700 & 40.5 & 6 & 30 \\
0.0060 & 0.700 & 35.5 & 6 & 60 \\
0.0040 & 0.400 & 6.5 & 4 & 80 \\
0.0040 & 0.700 & 25 & 3 & 40 \\
0.0040 & 1.000 & 19.5 & 4 & 30 \\
\hline
\end{tabular}




\section{Chapter 5}

\section{Conclusion and Future Work}

\subsection{Conclusion}

Conclusions in this work are given as follows:

(i) A robotic polishing system has been proposed and designed. The configuration of this polishing system is decomposed of an industrial articulated arm and a polishing end-effector. In particular, the articulated arm carries the end-effector to the prescribed locations with regulated velocity and regulated contact force. Cooperating with the robotic arm, the compliant polishing end-effector executes the surface material removal by rotation motion;

(ii) An off-line process planning for robotic polishing of uneven surface is accomplished. To obtain an even surface with the same thickness/roughness, polishing parameters are properly adapted in terms of the uneven situations of surface. To be more specific, using the proposed solution, the planned 
polishing process can fulfill the different desired material removal/roughness reduction for different surface areas;

(iii) The proposed solution combines NNW and GA. The polishing mechanisms are approximated by NNW and then GA integrated with NNW is employed to find out the optimum polishing parameters. Several experiments verify that the proposed planning solution can successfully achieve the expected performance: the desired surface material removal and the desired surface roughness improvement.

\subsection{Future Work}

Future works are given as follows:

(i) Research topics in the robotic MP planning for curved surfaces can be completed in the future work. Restricted by current measurement devices, in this case, the surface of curvature cannot be considered. Once the material removal and the roughness of curved surface can be accurately measured, we are able to further study how to do process planning to achieve ideal polishing performances. At that time, more polishing parameters can be discussed, such as the polishing tool path and the polishing tool orientation. In addition, more planning approaches can be utilized and compared, like particle swarm optimization, ant colony optimization and reinforcement learning optimization. 
(ii) Besides the robotic MP, the robotic electrochemical mechanical polishing (ECMP), this newly technology, can be studied. Compared to MP, ECMP can effectively achieve the lower surface roughness and the higher material removal rate. Furthermore, its integration with robotic techniques is capable of processing the workpiece of complex surface and huge size. Since the robotic ECMP involves many polishing parameters, like the applied voltage, the applied current, the pump frequency and the electrolyte $\mathrm{pH}$ value, the process planning of robotic ECMP becomes necessary to be developed. 


\section{Publications}

(1) A. E. K. Mohammad, J. Hong, and D. Wang, "Polishing of Uneven Surfaces Using Industrial Robots Based on Neural Network and Genetic Algorithm,” The International Journal of Advanced Manufacturing Technology, accepted for publication, manuscript number: JAMT-D-16-02825R1.

(2) A. E. K. Mohammad, J. Hong, and D. Wang, ’Design of a Force-controlled End-effector with Low-inertia Effect for Robotic Polishing using Macromini Robot Approach," Robotics and Computer-Integrated Manufacturing, accepted for publication, manuscript number: RCIM_2016_328_R1.

(3) J. Hong, A. E. K. Mohammad and D. Wang, "Improved Design of the Endeffector for Macro-Mini Robotic Polishing Systems," $20173^{\text {rd }}$ International Conference on Mechatronics and Robotics Engineering, to be published, manuscript number: M024. 


\section{Bibliography}

[1] Y. Shi, D. Zheng, L. Hu, Y. Wang, and L. Wang, "Nc polishing of aspheric surfaces under control of constant pressure using a magnetorheological torque servo," The International Journal of Advanced Manufacturing Technology, vol. 58, no. 9-12, pp. 1061-1073, 2012.

[2] M. Lee, S. Go, M. Lee, C. Jun, D. Kim, K. Cha, and J. Ahn, "A robust trajectory tracking control of a polishing robot system based on cam data," Robotics and Computer-Integrated Manufacturing, vol. 17, no. 1, pp. 177$183,2001$.

[3] D. Walker, D. Brooks, A. King, R. Freeman, R. Morton, G. McCavana, and S. W. Kim, "The precessions tooling for polishing and figuring flat, spherical and aspheric surfaces," Optics Express, vol. 11, no. 8, pp. 958964, 2003.

[4] F. J. Shiou and C. H. Chen, "Freeform surface finish of plastic injection mold by using ball-burnishing process," Journal of Materials Processing Technology, vol. 140, no. 1, pp. 248-254, 2003. 
[5] A. E. K. Mohammad and D. Wang, "Electrochemical mechanical polishing technology: recent developments and future research and industrial needs," The International Journal of Advanced Manufacturing Technology, vol. 5, no. 86, pp. 1909-1924, 2016.

[6] L. Guvenc and K. Srinivasan, "An overview of robot-assisted die and mold polishing with emphasis on process modeling," Journal of Manufacturing Systems, vol. 16, no. 1, p. 48, 1997.

[7] Y. Chen and Y. Song, "The development of a layer based machining system," Computer-Aided Design, vol. 33, no. 4, pp. 331-342, 2001.

[8] J. Pandremenos, C. Doukas, P. Stavropoulos, and G. Chryssolouris, "Machining with robots: a critical review," Proceedings of DET2011, 2011.

[9] M. Sabourin, F. Paquet, B. Hazel, J. Côté, and P. Mongenot, "Robotic approach to improve turbine surface finish," in 2010 1st International Conference on Applied Robotics for the Power Industry (CARPI). IEEE, 2010, pp. 1-6.

[10] B. Hazel, J. Côté, P. Mongenot, M. Sabourin, and F. Paquet, "Robotic polishing of turbine runners," in 2012 2nd International Conference on Applied Robotics for the Power Industry (CARPI). IEEE, 2012, pp. 50-51. 
[11] H. Huang, Z. Gong, X. Chen, and L. Zhou, "Robotic grinding and polishing for turbine-vane overhaul," Journal of materials processing technology, vol. 127, no. 2, pp. 140-145, 2002.

[12] E. Uhlmann, F. Heitmüller, M. Manthei, and S. Reinkober, “Applicability of industrial robots for machining and repair processes," Procedia CIRP, vol. 11, pp. 234-238, 2013.

[13] X. Pessoles and C. Tournier, "Automatic polishing process of plastic injection molds on a 5-axis milling center," Journal of materials processing technology, vol. 209, no. 7, pp. 3665-3673, 2009.

[14] R. T. Coelho, H. H. Rodella, V. F. Martins, J. Barba et al., "An investigation into the use of industrial robots for machining soft and low density materials with hsm technique," Journal of the Brazilian Society of Mechanical Sciences and Engineering, vol. 33, no. 3, pp. 343-350, 2011.

[15] E. Abele, M. Weigold, and S. Rothenbücher, "Modeling and identification of an industrial robot for machining applications," CIRP AnnalsManufacturing Technology, vol. 56, no. 1, pp. 387-390, 2007.

[16] F. Nagata, Y. Kusumoto, Y. Fujimoto, and K. Watanabe, "Robotic sanding system for new designed furniture with free-formed surface," Robotics and Computer-Integrated Manufacturing, vol. 23, no. 4, pp. 371-379, 2007. 
[17] F. Nagata, Y. Kusumoto, K. Watanabe, K. Tsuda, K. Yasuda, K. Yokoyama, M. Omoto, and H. Miyako, "Polishing robot for pet bottle molds using a learning-based hybrid position/force controller," in 5th Asian Control Conference, vol. 2. IEEE, 2004, pp. 914-921.

[18] C. Yueyan, Z. Ji, W. Bidou, and H. Shuang, "High precision fuzzy impedance control of free-form surfaces polishing robotic arm based on position control," in Proceedings, 2005 IEEE/ASME International Conference on Advanced Intelligent Mechatronics. IEEE, 2005, pp. 819-824.

[19] H. C. Song and J. B. Song, "Precision robotic deburring based on force control for arbitrarily shaped workpiece using cad model matching," International Journal of Precision Engineering and Manufacturing, vol. 14, no. 1, pp. 85-91, 2013.

[20] J. Dieste, A. Fernández, C. Javierre, and J. Santolaria, "Environmentally conscious polishing system based on robotics and artificial vision," Advances in Mechanical Engineering, vol. 7, no. 2, 2015.

[21] F. Tian, C. Lv, Z. Li, and G. Liu, "Modeling and control of robotic automatic polishing for curved surfaces," CIRP Journal of Manufacturing Science and Technology, vol. 14, pp. 55-64, 2016.

[22] A. E. K. Mohammad and D. Wang, "A novel mechatronics design of an electrochemical mechanical end-effector for robotic-based surface polish- 
ing," in 2015 IEEE/SICE International Symposium on System Integration (SII). IEEE, 2015, pp. 127-133.

[23] F. de Oliveira Campos, A. C. de Pina Filho, and A. C. de Pina, "Using robots to material removal processes," in Proceedings, the 9th Brazilian Conference on Dynamics Control and their Applications, 2010.

[24] F. Tian, Z. Li, C. Lv, and G. Liu, "Polishing pressure investigations of robot automatic polishing on curved surfaces," The International Journal of Advanced Manufacturing Technology, vol. 87, no. 1-4, pp. 639-646, 2016.

[25] F. Nagata, T. Hase, Z. Haga, M. Omoto, and K. Watanabe, “Cad/cam-based position/force controller for a mold polishing robot," Mechatronics, vol. 17, no. 4, pp. 207-216, 2007.

[26] H. Oktem, T. Erzurumlu, and F. Erzincanli, "Prediction of minimum surface roughness in end milling mold parts using neural network and genetic algorithm," Materials \& design, vol. 27, no. 9, pp. 735-744, 2006.

[27] G. Wang, Y. Wang, J. Zhao, and G. Chen, "Process optimization of the serial-parallel hybrid polishing machine tool based on artificial neural network and genetic algorithm," Journal of Intelligent manufacturing, vol. 23, no. 3 , pp. $365-374,2012$.

[28] P. Benardos and G. C. Vosniakos, "Prediction of surface roughness in cnc face milling using neural networks and taguchi's design of experiments," 
Robotics and Computer-Integrated Manufacturing, vol. 18, no. 5, pp. 343354, 2002.

[29] A. P. Markopoulos, D. E. Manolakos, and N. M. Vaxevanidis, "Artificial neural network models for the prediction of surface roughness in electrical discharge machining," Journal of Intelligent Manufacturing, vol. 19, no. 3, pp. 283-292, 2008.

[30] T. H. Sun, F. C. Tien, F.-C. Tien, and R. J. Kuo, "Automated thermal fuse inspection using machine vision and artificial neural networks," Journal of Intelligent Manufacturing, vol. 27, no. 3, pp. 639-651, 2016.

[31] C. Hartmann, D. Opritescu, and W. Volk, "An artificial neural network approach for tool path generation in incremental sheet metal freeforming," Journal of Intelligent Manufacturing, nov 2016, In press, DOI: 10.1007/s10845-016-1279-X.

[32] J. H. Holland, Adaptation in natural and artificial systems: an introductory analysis with applications to biology, control, and artificial intelligence. $\mathrm{U}$ Michigan Press, 1975.

[33] C. Moon, Y. Seo, Y. Yun, and M. Gen, "Adaptive genetic algorithm for advanced planning in manufacturing supply chain," Journal of Intelligent Manufacturing, vol. 17, no. 4, pp. 509-522, 2006. 
[34] N. Morad and A. Zalzala, "Genetic algorithms in integrated process planning and scheduling," Journal of Intelligent Manufacturing, vol. 10, no. 2, pp. 169-179, 1999.

[35] T. H. Hou, C. H. Su, and W. L. Liu, "Parameters optimization of a nanoparticle wet milling process using the taguchi method, response surface method and genetic algorithm," Powder Technology, vol. 173, no. 3, pp. 153-162, 2007.

[36] D. E. Golberg, "Genetic algorithms in search, optimization, and machine learning," Addion wesley, vol. 1989, p. 102, 1989.

[37] D. Xin, C. Hua. hua, and G. Wei. kang, "Neural network and genetic algorithm based global path planning in a static environment," Journal of Zhejiang University Science A, vol. 6, no. 6, pp. 549-554, 2005.

[38] J. Griffin, “The prediction of profile deviations when creep feed grinding complex geometrical features by use of neural networks and genetic programming with real-time simulation," The International Journal of Advanced Manufacturing Technology, vol. 74, no. 1-4, pp. 1-16, 2014.

[39] K. Li, S. Yan, W. Pan, and G. Zhao, "Warpage optimization of fiberreinforced composite injection molding by combining back propagation neural network and genetic algorithm," The International Journal of Advanced Manufacturing Technology, sep 2016, In press, DOI: 10.1007/s00170-016-9409-3. 
[40] C. Shen, L. Wang, and Q. Li, "Optimization of injection molding process parameters using combination of artificial neural network and genetic algorithm method," Journal of Materials Processing Technology, vol. 183, no. 2, pp. 412-418, 2007.

[41] O. Yilmaz, N. Gindy, and J. Gao, "A repair and overhaul methodology for aeroengine components," Robotics and Computer-Integrated Manufacturing, vol. 26, no. 2, pp. 190-201, 2010.

[42] A. Ranganathan, "The levenberg-marquardt algorithm," Tutoral on LM algorithm, pp. 1-5, 2004.

[43] D. W. Marquardt, “An algorithm for least-squares estimation of nonlinear parameters," Journal of the society for Industrial and Applied Mathematics, vol. 11, no. 2, pp. 431-441, 1963.

[44] J. Huissoon, F. Ismail, A. Jafari, and S. Bedi, "Automated polishing of die steel surfaces," The international journal of advanced manufacturing technology, vol. 19, no. 4, pp. 285-290, 2002.

[45] Y. Chen and F. Dong, "Robot machining: recent development and future research issues," The International Journal of Advanced Manufacturing Technology, vol. 66, no. 9-12, pp. 1489-1497, 2013. 